\title{
BMJ open Effects of exenatide and liraglutide on heart rate, blood pressure and body weight: systematic review and meta-analysis
}

\author{
Louise E Robinson, ${ }^{1}$ Tim A Holt, ${ }^{1,2}$ Karen Rees, ${ }^{1}$ Harpal S Randeva, ${ }^{1}$ \\ Joseph P O'Hare ${ }^{1}$
}

To cite: Robinson LE, Holt TA, Rees K, et al. Effects of exenatide and liraglutide on heart rate, blood pressure and body weight: systematic review and meta-analysis. BMJ Open 2013;3:e001986. doi:10.1136/bmjopen-2012001986

- Prepublication history for this paper are available online. To view these files please visit the journal online (http://dx.doi.org/10.1136/ bmjopen-2012-001986).

Received 28 August 2012 Revised 15 November 2012 Accepted 3 December 2012

This final article is available for use under the terms of the Creative Commons Attribution Non-Commercial 2.0 Licence; see http://bmjopen.bmj.com

${ }^{1}$ Warwick Medical School, University of Warwick, Coventry, UK

${ }^{2}$ Department of Primary Care Health Sciences, University of Oxford, Oxford, UK

Correspondence to Dr Tim A Holt; tim.holt@phc.ox.ac.uk

\section{ABSTRACT}

Objectives: To synthesise current evidence for the effects of exenatide and liraglutide on heart rate, blood pressure and body weight.

Design: Meta-analysis of available data from randomised controlled trials comparing Glucagon-like peptide-1 (GLP-1) analogues with placebo, active antidiabetic drug therapy or lifestyle intervention.

Participants: Patients with type 2 diabetes. Outcome measures: Weighted mean differences between trial arms for changes in heart rate, blood pressure and body weight, after a minimum of 12-week follow-up.

Results: 32 trials were included. Overall, GLP-1 agonists increased the heart rate by 1.86 beats/min (bpm) $(95 \% \mathrm{Cl} 0.85$ to 2.87$)$ versus placebo and $1.90 \mathrm{bpm}$ (1.30 to 2.50) versus active control. This effect was more evident for liraglutide and exenatide long-acting release than for exenatide twice daily. GLP-1 agonists decreased systolic blood pressure by $-1.79 \mathrm{~mm} \mathrm{Hg}(-2.94$ to -0.64$)$ and $-2.39 \mathrm{~mm} \mathrm{Hg}$ $(-3.35$ to -1.42$)$ compared to placebo and active control, respectively. Reduction in diastolic blood pressure failed to reach statistical significance $(-0.54 \mathrm{~mm} \mathrm{Hg}(-1.15$ to 0.07$)$ vs placebo and $-0.50 \mathrm{~mm} \mathrm{Hg}(-1.24$ to 0.24$)$ vs active control). Body weight decreased by $-3.31 \mathrm{~kg}(-4.05$ to -2.57$)$ compared to active control, but by only $-1.22 \mathrm{~kg}$ $(-1.51$ to -0.93$)$ compared to placebo.

Conclusions: GLP-1 analogues are associated with a small increase in heart rate and modest reductions in body weight and blood pressure. Mechanisms underlying the rise in heart rate require further investigation.

\section{INTRODUCTION}

In contrast to the weight-increasing effects of several traditional antidiabetic drug classes, ${ }^{1}$ Glucagon-like peptide-1 (GLP-1) analogues have been shown to reduce both body weight and blood pressure. ${ }^{2}$ The mechanisms producing weight loss have been extensively

\section{ARTICLE SUMMARY}

Article focus

- Glucagon-like peptide-1 (GLP-1) agonists are increasingly used in the management of type 2 diabetes, but their long-term cardiovascular safety is not yet confirmed.

- These agents are known to reduce body weight and blood pressure, but are also associated with an elevation in heart rate that has not previously been quantified.

Key messages

- Our analysis confirms the weight and blood pressure reducing effects of liraglutide and exenatide, and reports a small rise in heart rate.

- The weight-reducing effects are substantially greater when compared with active control treatments than placebo, as alternative treatment options may promote weight gain.

- Heart rate rises were more evident for liraglutide than exenatide, and for exenatide long-acting release than exenatide twice daily.

Strengths and limitations

- We included unpublished data obtained from pharmaceutical companies, enabling the effects of GLP-1 agonists on heart rate to be quantified for the first time by meta-analysis.

- Our analysis is limited by significant heterogeneity between studies, and suggests the need for more detailed investigation using more accurate measurements of heart rate than those typically used in clinical practice.

investigated and involve improved satiety and reduced calorie ingestion, both through effects on the central nervous system and through delayed gastric emptying. ${ }^{3-6}$ Those leading to reduced blood pressure are less adequately understood, but this effect has been shown to occur as early as 2 weeks after the start of the therapy, preceding significant weight loss, suggesting that a direct 
hypotensive effect is at least partly responsible. $^{7}$ Experimental studies of GLP-1 analogues have also reported direct effects on blood pressure, possibly via interaction with the autonomic nervous system. ${ }^{8} 9$

While a number of studies have reported heart rate increases, the associated mechanisms are unknown, and this effect is often dismissed as clinically unimportant. Given the safety implications attributed to raised heart rate in other contexts, ${ }^{10-13}$ there is a surprising lack of concern over its possible implications in this setting. A recent review of liraglutide by Bode acknowledges the effect, ${ }^{14}$ but a meta-analysis on the safety of incretinbased therapies published in 2010 did not mention heart rate, ${ }^{15}$ and neither did an overview of the LEAD trials of liraglutide by Blonde and Russell-Jones. ${ }^{16}$ A large nationwide audit of exenatide designed by the Association of British Clinical Diabetologists (ABCD) did not include heart rate as an outcome, despite citing evidence for the effect in the main published report. ${ }^{17}$ A subsequent (ongoing) $\mathrm{ABCD}$ audit of liraglutide also aims to identify unknown safety issues but has similarly omitted heart rate from the protocol. ${ }^{18}$

GLP-1 analogues are an expanding drug class with the recent development of longer acting agents including the once weekly (LAR) form of exenatide, Bydureon. This drug has recently obtained approval from the National Institute for Health and Clinical Excellence for use in type 2 diabetes, and its use is likely to increase. ${ }^{19}$ A review of trial data from five long-acting GLP-1 agonists (exenatide once weekly, taspoglutide, albiglutide, LY2189265 and CJC-1134-PC) concluded that they were more likely than shorter acting formulations to raise the heart rate. ${ }^{20} \mathrm{~A}$ more recently published study of the longacting GLP-1 agent PF-04603629 reported a substantial rise in the heart rate (a mean increase of $23 \mathrm{bpm}$ at $24 \mathrm{~h}$ after injection of the higher dose studied), together with a rise in the diastolic blood pressure. ${ }^{21}$

While there is no evidence to date that these agents (short-acting or long-acting) increase cardiovascular event rates, safety data are limited by short follow-up duration. $^{22}$ A longer term follow-up is underway but will take a number of years to complete.

We aimed to identify and synthesise all available heart rate data from both published and unpublished sources to quantify the effect of GLP-1 analogues on heart rate, as well as that on blood pressure and body weight.

\section{METHODS}

\section{Literature searches}

The following resources were systematically searched to identify completed, new or ongoing controlled trials of liraglutide or exenatide: Clinical Trials Gov (http://www. clinicaltrials.gov); Entertrials.co.uk; Clinicaltrialssearch.

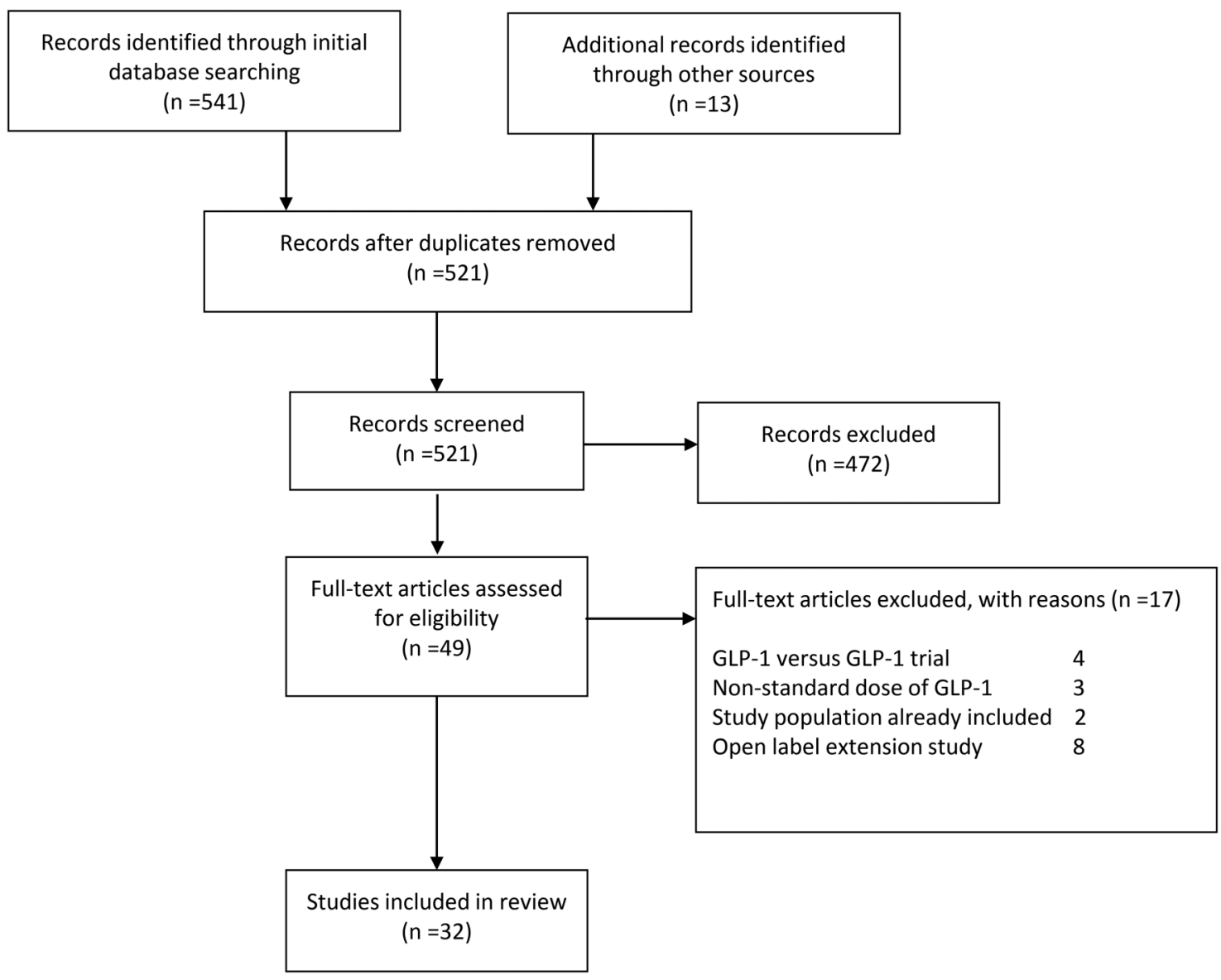

Figure 1 PRISMA flow diagram. GLP-1, glucagon-like peptide-1. 
Table 1 Characteristics of included studies

\begin{tabular}{|c|c|c|c|c|c|c|c|c|c|}
\hline Study & Comparisons & $\begin{array}{l}\text { Duration } \\
\text { (weeks) }\end{array}$ & $\begin{array}{l}\text { Study } \\
\text { population/ } \\
\text { ethnicity }\end{array}$ & Country & $\begin{array}{l}\text { Body weight } \\
\text { groups } \\
\text { included }\end{array}$ & $\begin{array}{l}\text { Balanced } \\
\text { male/female? }\end{array}$ & $\begin{array}{l}\text { Mean } \\
\text { age }\end{array}$ & $\begin{array}{l}\text { Standardised } \\
\text { diet/exercise }\end{array}$ & Background OAD \\
\hline Apovian et $a{ }^{42}$ & EX/PLAC & 24 & MR & US & OW & $>60 \% \mathrm{~F}$ & 54.8 & $Y$ & MET and/or SU \\
\hline Barnett et $a{ }^{43}$ & $E X / I G$ & 16 & MR & Multinational & $\mathrm{N} / \mathrm{OW} / \mathrm{OB}$ & $\mathrm{Y}$ & 54.9 & $\mathrm{~N}$ & MET or SU \\
\hline $\begin{array}{l}\text { Bergenstal } \\
\text { et } a{ }^{44}\end{array}$ & EX/BIAsp & 24 & MR & US & $\mathrm{N} / \mathrm{OW}$ & $\mathrm{Y}$ & 52.6 & $\mathrm{~N}$ & MET and SU \\
\hline $\begin{array}{l}\text { Bergenstal } \\
\text { et } a f^{55}\end{array}$ & $\begin{array}{l}\text { EX LAR vs } \\
\text { PIO } \\
\text { EX LAR vs } \\
\text { SITA }\end{array}$ & 26 & MR & Multinational & $\mathrm{N} / \mathrm{OW} / \mathrm{OB}$ & $\mathrm{Y}$ & 52.3 & $\mathrm{~N}$ & MET \\
\hline Buse et $a l^{46}$ & EX/PLAC & 30 & MR & US & OW/OB & $60 \% \mathrm{M}$ & 55.3 & $\mathrm{~N}$ & SU \\
\hline Buse et $a{ }^{47}$ & $\begin{array}{l}\mathrm{IG}+\mathrm{EX} / \mathrm{IG} \\
+\mathrm{PLAC}\end{array}$ & 30 & MR & Multinational & N/OW/OB & $\mathrm{Y}$ & 59.0 & $\mathrm{~N}$ & MET or PIO \\
\hline Davies et $a /^{48}$ & $E X / I G$ & 26 & MR & GB & OW/OB & $>60 \% \mathrm{M}$ & 56.5 & $\mathrm{~N}$ & $\begin{array}{l}\text { Two or three OADS: } \\
\text { MET, SU, or TZD }\end{array}$ \\
\hline $\begin{array}{l}\text { DeFronzo } \\
\text { et } a{ }^{49}\end{array}$ & EX/PLAC & 30 & MR & US & OW/OB & $\mathrm{Y}$ & 53.0 & $\mathrm{~N}$ & MET \\
\hline $\begin{array}{l}\text { DeFronzo } \\
\text { et } a{ }^{50}\end{array}$ & EX vs ROSI & 20 & MR & US & OW/OB & $\mathrm{Y}$ & 56.0 & $\mathrm{~N}$ & MET \\
\hline Derosa et $a^{51}$ & EX/GLIB & 52 & W & IT & OW/OB & $Y$ & 56.5 & $Y$ & MET \\
\hline Derosa et $a^{p^{2}}$ & EX/GLIM & 52 & CAUC & IT & OW/OB & $\mathrm{Y}$ & 55.5 & $\mathrm{Y}$ & MET \\
\hline Diamant et $a^{53}$ & EX LAR/IG & 26 & MR & Multinational & OW/OB & $\mathrm{Y}$ & 58.0 & $\mathrm{~N}$ & MET \\
\hline Gallwitz et $a^{54}$ & EX/BIAsp & 26 & MR & GER & OW/OB & Not reported & 57.0 & $\mathrm{~N}$ & MET \\
\hline Gallwitz et $a^{55}$ & EX/GLIM & $\begin{array}{l}\text { Up to } \\
4.5 \text { years }\end{array}$ & MR & Multinational & $\mathrm{OW} / \mathrm{OB}$ & $\mathrm{Y}$ & 56.0 & $\mathrm{~N}$ & MET \\
\hline Gao et $a^{56}$ & EX/PLAC & 12 & $\mathrm{C} / \mathrm{I} / \mathrm{K} / \mathrm{T}$ & Multinational & N/OW/OB & $\mathrm{Y}$ & 54.0 & $\mathrm{~N}$ & MET and/or SU \\
\hline Garber et $a^{p^{7}}$ & LIR/GLIM & 52 & MR & US/MEX & $\mathrm{N} / \mathrm{OW} / \mathrm{OB}$ & $\mathrm{Y}$ & 53.0 & $\mathrm{~N}$ & $\begin{array}{l}\text { Nil-previous OAD } \\
\text { withdrawn }\end{array}$ \\
\hline Gill et $a^{58}$ & EX/PLAC & 12 & MR & CAN/NL & OW/OB & $Y$ & 55.6 & $\mathrm{~N}$ & MET and/or TZD \\
\hline Heine et $a^{59}$ & $E X / I G$ & 26 & MR & Multinational & OW/OB & $\mathrm{Y}$ & 58.9 & $\mathrm{~N}$ & MET and SU \\
\hline Kadowaki et a ${ }^{60}$ & EX/PLAC & 12 & $J P$ & $\mathrm{JP}$ & N/OW/OB & $>60 \% \mathrm{M}$ & 60.3 & $\mathrm{~N}$ & $\begin{array}{l}\text { SU, with or without } \\
\text { either BG or TZD }\end{array}$ \\
\hline Kendall et $a^{61}$ & EX/PLAC & 30 & MR & US & OW/OB & $Y$ & 55.3 & $Y$ & MET and SU \\
\hline Kim et $a^{62}$ & EX LAR/PLAC & 15 & MR & US & OW/OB & $60 \% \mathrm{M}$ & 53.7 & $Y$ & MET \\
\hline Liutkus et $a^{63}$ & EX/PLAC & 26 & MR & Multinational & OW/OB & $\mathrm{Y}$ & 54.7 & $\mathrm{~N}$ & $\begin{array}{l}\text { TZD with or without } \\
\text { MET }\end{array}$ \\
\hline Marre et $a \wp^{4}$ & $\begin{array}{l}\text { LIR/PLAC/ } \\
\text { ROSI }\end{array}$ & 26 & MR & Multinational & $\mathrm{N} / \mathrm{OW} / \mathrm{OB}$ & $Y$ & 56.0 & $\mathrm{~N}$ & SU \\
\hline Moretto et $a^{65}$ & EX/PLAC & 24 & MR & Multinational & OW/OB & $\mathrm{Y}$ & 54.0 & $Y$ & DRUG NAIVE \\
\hline Nauck et $a^{66}$ & EX/PIA & 52 & MR & Multinational & $\mathrm{OW} / \mathrm{OB}$ & $\mathrm{Y}$ & 58.5 & $\mathrm{~N}$ & SU and MET \\
\hline Nauck et a $f^{7}$ & $\begin{array}{l}\text { LIR/GLIM/ } \\
\text { PLAC }\end{array}$ & 26 & MR & Multinational & N/OW/OB & $\mathrm{Y}$ & 56.7 & $\mathrm{~N}$ & MET \\
\hline
\end{tabular}


Table 1 Continued

\begin{tabular}{|c|c|c|c|c|c|c|c|c|c|}
\hline Study & Comparisons & $\begin{array}{l}\text { Duration } \\
\text { (weeks) }\end{array}$ & $\begin{array}{l}\text { Study } \\
\text { population/ } \\
\text { ethnicity }\end{array}$ & Country & $\begin{array}{l}\text { Body weight } \\
\text { groups } \\
\text { included }\end{array}$ & $\begin{array}{l}\text { Balanced } \\
\text { male/female? }\end{array}$ & $\begin{array}{l}\text { Mean } \\
\text { age }\end{array}$ & $\begin{array}{l}\text { Standardised } \\
\text { diet/exercise }\end{array}$ & Background OAD \\
\hline Pratley et $a^{68}$ & LIR/SIT & 26 & MR & Multinational & N-OW-OB & $Y$ & 55.3 & $\mathrm{~N}$ & MET \\
\hline $\begin{array}{l}\text { Russell-Jones } \\
\text { et af9 }\end{array}$ & LIR/IG/PLAC & 26 & MR & Multinational & N/OW/OB & $Y$ & 57.5 & $\mathrm{~N}$ & MET and SU \\
\hline $\begin{array}{l}\text { Russell-Jones } \\
\text { et } a l^{00}\end{array}$ & $\begin{array}{c}\text { EX LAR/MET } \\
\text { EX LAR/PIO } \\
\text { EX LAR/SITA }\end{array}$ & 26 & MR & Multinational & N/OW/OB & $Y$ & 54.0 & $\mathrm{~N}$ & DRUG NAIVE \\
\hline Yang et $a l^{71}$ & LIR/GLIM & 16 & $\mathrm{C} / \mathrm{K} / \mathrm{I}$ & Multinational & N/OW/OB & $Y$ & 53.3 & $\mathrm{~N}$ & MET \\
\hline Zinman et $a l^{73}$ & LIR/PLAC & 26 & MR & US/CAN & $\mathrm{N} / \mathrm{OW} / \mathrm{OB}$ & $Y$ & 55.0 & $\mathrm{~N}$ & MET and ROSI \\
\hline
\end{tabular}

BG, Biguanide; BIAsp, biphasic insulin aspart; C, Chinese; CAN, Canada; CAUC, Caucasian; EX LAR, exenatide long-acting release; EX, exenatide; GB, Great Britain; GER, Germany; GLIB, glibenclamide; GLIM, glimepiride; I, Indian; IG, insulin glargine; IT, Italy; JP, Japan; JP, Japanese; K, Korean; LIR, liraglutide; MET, metformin; MEX, Mexico; MR, Multiracial; N, normal weight; $\mathrm{NL}$, the Netherlands; OAD, oral antidiabetic drug; OB, obese; OW, overweight; PIO, pioglitazone; PLAC, placebo; ROSI, rosiglitazone; SITA, sitagliptin; T, Taiwanese; US, the USA; W, White.

Table 2 Risk of bias across included studies

\begin{tabular}{|c|c|c|c|c|c|c|c|c|c|}
\hline No. & Study & $\mathbf{A}$ & $\mathbf{B}$ & $\mathbf{C}$ & $\mathbf{D}$ & $E$ & $\mathbf{F}$ & $\mathbf{G}$ & Comments \\
\hline 1 & Apovian et $a f^{12} \dagger$ & 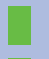 & 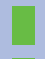 & 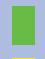 & 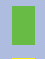 & 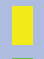 & 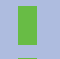 & 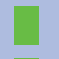 & Greater than $20 \%$ attrition \\
\hline 2 & Barnett et $a{ }^{43 *} \dagger$ & 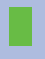 & 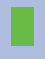 & & 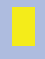 & 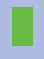 & 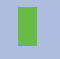 & 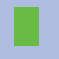 & Open label cross-over study \\
\hline 3 & Bergenstal et $a f^{44_{*}} \dagger$ & 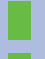 & 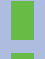 & - & 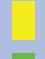 & 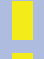 & $=$ & 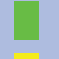 & Open label. Greater than $20 \%$ attrition and higher attrition in the exenatide group \\
\hline 4 & Bergenstal et $a f^{45} \dagger$ & 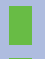 & 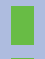 & 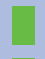 & 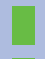 & 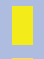 & 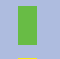 & 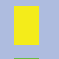 & Greater than $20 \%$ attrition. Outcome assessors unblinded after finalisation of the analysis plan \\
\hline 5 & Buse et $a t^{46}$ & 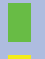 & 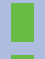 & 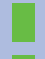 & - & - & - & - & Greater than $20 \%$ attrition. Higher attrition in the placebo arm \\
\hline 6 & Buse et $a{ }^{47} \dagger$ & 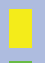 & 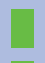 & 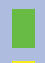 & 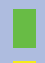 & 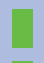 & 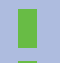 & - & Groups not balanced for sex and concomitant medication \\
\hline 7 & Davies et $a f^{48 *}$ & 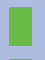 & 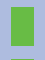 & 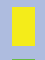 & . & 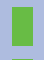 & 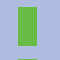 & 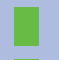 & Open label \\
\hline 8 & DeFronzo et a ${ }^{49}$ & 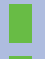 & 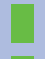 & 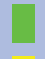 & 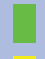 & 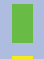 & 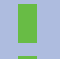 & 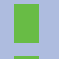 & \\
\hline 9 & DeFronzo et $a^{50_{*}} \dagger$ & 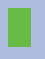 & 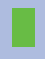 & ( & 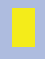 & ( & 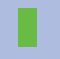 & 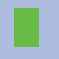 & Open label. Greater than $20 \%$ attrition \\
\hline 10 & Derosa et $a^{\lceil 1}$ & 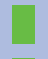 & 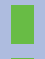 & 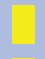 & 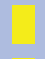 & 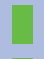 & 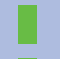 & 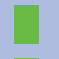 & Single blind \\
\hline 11 & Derosa et $a^{\Gamma^{2}} \emptyset$ & 咀 & L & 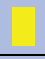 & 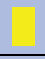 & 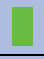 & 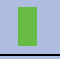 & 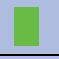 & Single blind \\
\hline
\end{tabular}




\begin{tabular}{|c|c|c|c|c|c|c|c|c|}
\hline No. & Study & A & B & C & D & E $\mathbf{F}$ & $F \mathbf{G}$ & Comments \\
\hline 12 & Diamant et $a^{53 *} \ddagger$ & a & 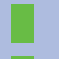 & & I & 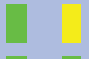 & 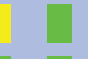 & Open label. Higher attrition in the exenatide arm \\
\hline 13 & Gallwitz et $a^{54 *}$ & 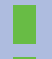 & 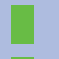 & F & L & 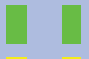 & 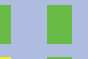 & Open label \\
\hline 14 & Gallwitz et $a^{55 *}$ & a & 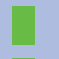 & - & 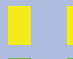 & (1) & - & Open label. Greater than $20 \%$ attrition. Higher attrition in the exenatide arm \\
\hline 15 & Gao et $a^{56} \dagger$ & a & 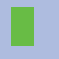 & 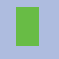 & 1 & 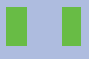 & 1 & \\
\hline 16 & Garber et $a^{{ }^{7}} \dagger$ & a & $=$ & 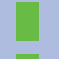 & 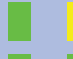 & $=$ & 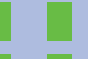 & Greater than $20 \%$ attrition \\
\hline 17 & Gill et $a^{58}$ & 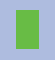 & 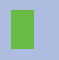 & 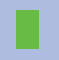 & 1 & I & 1 & \\
\hline 18 & Heine et $a^{59 *}$ & - & 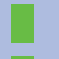 & - & - & 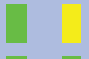 & - & Open label. Higher attrition in the exenatide arm \\
\hline 19 & Kadowaki et $a^{60} \S$ & a & 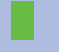 & a & a & 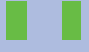 & 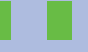 & \\
\hline 20 & Kendall et a ${ }^{61}$ & 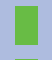 & 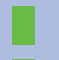 & 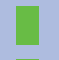 & 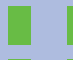 & 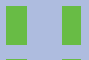 & 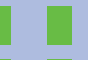 & \\
\hline 21 & Kim et $a^{62}$ & a & 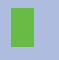 & 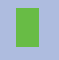 & 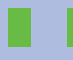 & 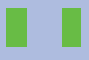 & 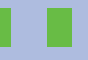 & \\
\hline 22 & Liutkus et $a^{{ }^{3} \S}$ & 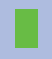 & 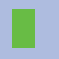 & 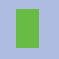 & 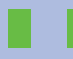 & 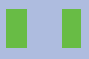 & - & \\
\hline 23 & Marre et $a^{64}$ & 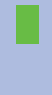 & 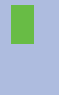 & $\square$ & 1 & 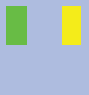 & 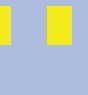 & $\begin{array}{l}\text { Higher attrition in the placebo arm. Restriction of glimipiride and rosiglitazone in some countries precluded } \\
\text { maximal dose regimes }\end{array}$ \\
\hline 24 & Moretto et $a^{65}$ & 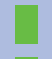 & 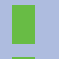 & - & 1 & 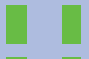 & 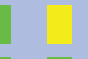 & Diet and exercise regimes not standardised \\
\hline 25 & Nauck et $a^{66 *} \dagger$ & 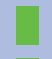 & 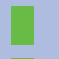 & 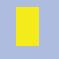 & L & 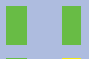 & 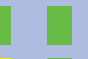 & Open label \\
\hline 26 & Nauck et $a^{67} \dagger$ & 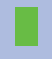 & 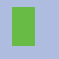 & 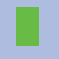 & 1 & 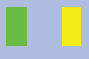 & - & Higher attrition in the liraglutide $1.8 \mathrm{mg}$ and placebo arms \\
\hline 27 & Pratley et $a^{68 *}$ & 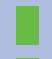 & 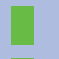 & 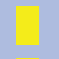 & 1 & 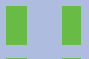 & 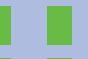 & Open label, but statistician was masked to the allocation \\
\hline 28 & Russell-Jones et $a^{69^{*}} \dagger$ & a & 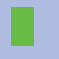 & 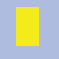 & I & 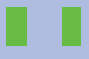 & 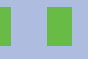 & Insulin glargine arm-open label \\
\hline 29 & Russell-Jones et $a l^{70} \dagger$ & 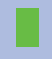 & 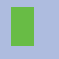 & 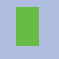 & 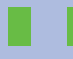 & 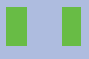 & a & \\
\hline 30 & Yang et $a l^{71}$ & 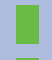 & 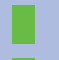 & 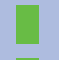 & 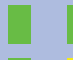 & 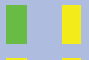 & - & Higher attrition in the liraglutide groups \\
\hline 31 & Zinman et $a l^{72}$ & 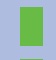 & - & 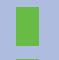 & 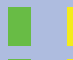 & T & a & Greater than $20 \%$ attrition. Higher attrition in the exenatide group \\
\hline 32 & Zinman et al ${ }^{73}$ & 1 & L & L & L & t & 1 & Greater than $20 \%$ attrition. Higher attrition in the placebo group \\
\hline \multicolumn{9}{|c|}{$\begin{array}{l}\text { Included studies were assessed using the Cochrane Risk of Bias Tool for factors which may cause bias in the trial outcomes and subsequent evaluation by meta-analysis: (A) randomisation, (B) } \\
\text { allocation concealment, (C) blinding of participants/investigators/sponsors, (D) blinding outcome assessment, (E) incomplete outcome data, (F) selective outcome reporting and (G) other bias. } \\
\text { *Open label. } \\
\text { tMethod of randomisation/allocation concealment consisted of a computer random-number generator and voice-response or telephone system. } \\
\text { fPermuted block randomisation. } \\
\text { \&Randomised according to the baseline biochemical values or background pharmacological agent. } \\
\text { IRandomised according to coded envelopes designed by a statistician } \\
\text { high risk; low risk; unclear risk. }\end{array}$} \\
\hline
\end{tabular}


org; Centerwatch; Drugsontrial; WebMD; MEDLINE (from 1960); EMBASE (from 1960); Cochrane Library Central Register of Controlled Trials (CENTRAL). We used a search strategy to capture 'exenatide', 'liraglutide' or 'glucagon-like peptide-1' in any field, limited to 'Randomised Controlled Trial', 'Clinical Trial' or 'Controlled Clinical Trial'. Conference proceedings (British Endocrinology Society, Diabetes UK, European Association for the Study of Diabetes) and websites (American Diabetes Association, Federal Drug Agency and
European Medicines Agency) were examined, and the reference lists of trials, meta-analyses and reviews were searched for further studies. Novo Nordisk and Amylin Pharmaceuticals were contacted directly to request unpublished data. The review is up to date at July 2012.

Inclusion and exclusion criteria

A. Participants: We only included trials involving participants with type 2 diabetes.

\section{a Liraglutide versus placebo}

$$
\text { Experimental }
$$

Control

Mean Difference

Mean Difference

Study or Subgroup Mean [bpm] SD [bpm] Total Mean [bpm] SD [bpm] Total Weight $\quad$ IV, Random, 95\% Cl [bpm] IV, Random, 95\% Cl [bpm] 17.1.1 Liraglutide $1.2 \mathrm{mg}$ vs placebo

\begin{tabular}{|c|c|c|c|c|c|c|c|c|}
\hline Marre, 2009 & 2.49 & 8.9 & 228 & -1.55 & 8.3 & 57 & $13.2 \%$ & $4.04[1.60,6.48]$ \\
\hline Nauck 2009 & 2.13 & 8.9 & 240 & 1.06 & 8.3 & 60 & $13.5 \%$ & $1.07[-1.31,3.45]$ \\
\hline Zinman 2009 & 2.4 & 8.9 & 178 & 0.25 & 8.3 & 89 & $14.7 \%$ & $2.15[-0.01,4.31]$ \\
\hline Subtotal $(95 \% \mathrm{Cl})$ & & & 646 & & & 206 & $41.4 \%$ & $2.39[0.75,4.03]$ \\
\hline \multicolumn{9}{|c|}{ Heterogeneity: $\mathrm{Tau}^{2}=0.69 ; \mathrm{Chi}^{2}=2.98, \mathrm{df}=2(\mathrm{P}=0.23) ; \mathrm{I}^{2}=33 \%$} \\
\hline \multicolumn{9}{|c|}{ Test for overall effect: $Z=2.86(P=0.004)$} \\
\hline \multicolumn{9}{|c|}{ 17.1.2 Liraglutide $1.8 \mathrm{mg}$ vs placebo } \\
\hline Marre, 2009 & 4.54 & 8.4 & 234 & -1.55 & 8.3 & 57 & $13.4 \%$ & $6.09[3.68,8.50]$ \\
\hline Nauck 2009 & 2.07 & 8.4 & 242 & 1.06 & 8.3 & 61 & $13.7 \%$ & $1.01[-1.33,3.35]$ \\
\hline Russell- Jones, 2009 & 2.21 & 8.4 & 230 & 0.19 & 8.3 & 114 & $16.6 \%$ & $2.02[0.15,3.89]$ \\
\hline Zinman 2009 & 3.12 & 8.4 & 178 & 0.25 & 8.3 & 88 & $15.0 \%$ & $2.87[0.74,5.00]$ \\
\hline Subtotal $(95 \% \mathrm{Cl})$ & & & 884 & & & 320 & $58.6 \%$ & $2.95[0.96,4.94]$ \\
\hline
\end{tabular}

Heterogeneity: $\mathrm{Tau}^{2}=2.90 ; \mathrm{Chi}^{2}=10.09, \mathrm{df}=3(\mathrm{P}=0.02) ; 1^{2}=70 \%$

Test for overall effect: $Z=2.90(P=0.004)$

Total $(95 \% \mathrm{Cl})$

1530

$526 \quad 100.0 \%$

Heterogeneity: $\mathrm{Tau}^{2}=1.58 ; \mathrm{Chi}^{2}=13.34, \mathrm{df}=6(\mathrm{P}=0.04) ; \mathrm{I}^{2}=55 \%$

Test for overall effect: $Z=4.22(P<0.0001)$

Test for subgroup differences: $\mathrm{Chi}^{2}=0.18, \mathrm{df}=1(\mathrm{P}=0.67), \mathrm{I}^{2}=0 \%$

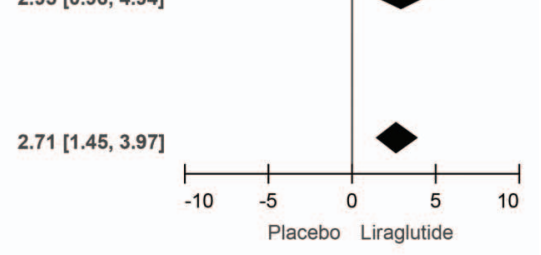

\section{b Liraglutide versus active control}

\begin{tabular}{|c|c|c|c|c|c|c|c|c|c|c|c|}
\hline \multirow[b]{2}{*}{ Study or Subgroup } & \multicolumn{3}{|c|}{ Experimental } & \multicolumn{3}{|c|}{ Control } & \multicolumn{2}{|r|}{ Mean Difference } & \multicolumn{3}{|c|}{ Mean Difference } \\
\hline & Mean [bpm] & SD [bpm] & Total & Mean [bpm] & SD [bpm] & Total & Weight & IV, Random, 95\% Cl [bpm] & IV, Random, 9 & $95 \% \mathrm{Cl}$ [bpm] & \\
\hline \multicolumn{12}{|c|}{ 17.2.3 Liraglutide $1.2 \mathrm{mg}$ vs active control } \\
\hline Garber, 2009 & 3.17 & 8.9 & 251 & 0.43 & 8.3 & 124 & $10.7 \%$ & $2.74[0.91,4.57]$ & & & \\
\hline Marre, 2009 & 2.49 & 8.9 & 228 & 1.09 & 8.3 & 116 & $10.2 \%$ & $1.40[-0.50,3.30]$ & & & \\
\hline Nauck 2009 & 2.13 & 8.9 & 240 & 0.58 & 8.3 & 121 & $10.5 \%$ & $1.55[-0.31,3.41]$ & & & \\
\hline Pratley, 2010 & 2.32 & 8.9 & 225 & -0.64 & 8.3 & 209 & $12.6 \%$ & $2.96[1.34,4.58]$ & & & \\
\hline Subtotal $(95 \% \mathrm{Cl})$ & & & 944 & & & 570 & $44.0 \%$ & $2.23[1.34,3.13]$ & & & \\
\hline \multicolumn{12}{|c|}{ Heterogeneity: $\mathrm{Tau}^{2}=0.00 ; \mathrm{Chi}^{2}=2.33, \mathrm{df}=3(\mathrm{P}=0.51) ; \mathrm{l}^{2}=0 \%$} \\
\hline \multicolumn{12}{|c|}{ Test for overall effect: $Z=4.89(P<0.00001)$} \\
\hline \multicolumn{12}{|c|}{ 17.2.4 Liraglutide $1.8 \mathrm{mg}$ vs active control } \\
\hline Garber, 2009 & 1.6 & 8.4 & 246 & 0.43 & 8.3 & 124 & $11.0 \%$ & $1.17[-0.63,2.97]$ & & & \\
\hline Marre, 2009 & 4.54 & 8.4 & 234 & 1.09 & 8.3 & 116 & $10.5 \%$ & $3.45[1.60,5.30]$ & & & \\
\hline Nauck 2009 & 2.07 & 8.4 & 242 & 0.58 & 8.3 & 121 & $10.8 \%$ & $1.49[-0.33,3.31]$ & & & \\
\hline Pratley, 2010 & 3.94 & 8.4 & 221 & -0.64 & 8.3 & 110 & $10.1 \%$ & $4.58[2.67,6.49]$ & & & \\
\hline Russell- Jones, 2009 & 2.21 & 8.4 & 230 & -0.75 & 8.3 & 232 & $13.6 \%$ & $2.96[1.44,4.48]$ & & & \\
\hline Subtotal $(95 \% \mathrm{Cl})$ & & & 1173 & & & 703 & $56.0 \%$ & $2.72[1.53,3.90]$ & & & \\
\hline \multicolumn{12}{|c|}{ Heterogeneity: $\mathrm{Tau}^{2}=1.01 ; \mathrm{Chi}^{2}=8.96, \mathrm{df}=4(P=0.06) ; \mathrm{I}^{2}=55 \%$} \\
\hline \multicolumn{12}{|c|}{ Test for overall effect: $Z=4.49(P<0.00001)$} \\
\hline Total $(95 \% \mathrm{Cl})$ & & & 2117 & & & 1273 & $100.0 \%$ & $2.49[1.77,3.21]$ & & & \\
\hline \multicolumn{9}{|c|}{ Heterogeneity: $\operatorname{Tau}^{2}=0.40 ; \mathrm{Chi}^{2}=11.88, \mathrm{df}=8(\mathrm{P}=0.16) ; \mathrm{I}^{2}=33 \%$} & & - & \\
\hline \multicolumn{9}{|c|}{ Test for overall effect: $Z=6.75(P<0.00001)$} & -5 & 5 & 10 \\
\hline
\end{tabular}

Figure 2 Effect of liraglutide on heart rate in patients with type 2 diabetes. 
B. Study designs: We included all randomised trials with a minimum follow-up of 12 weeks. We excluded 'open-label' extension studies of phase 3 trials.
C. Interventions: Trials of liraglutide (1.2 or $1.8 \mathrm{mg}$ daily), exenatide ( 5 or $10 \mu \mathrm{g}$ twice daily) or exenatide LAR, either alone or in combination with an

\section{(a) Exenatide versus placebo}

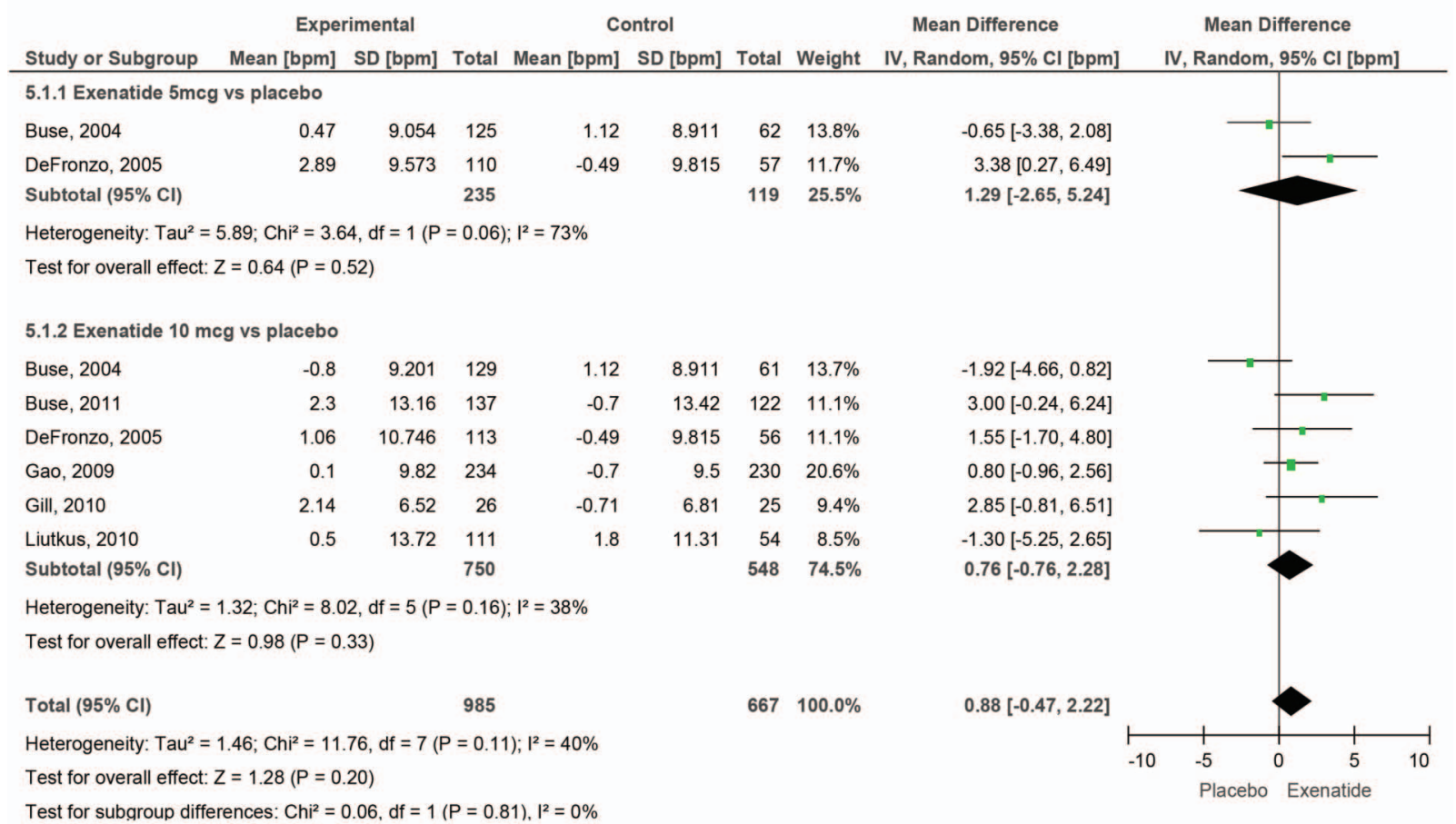

\section{(b) Exenatide versus active control}

\begin{tabular}{|c|c|c|c|c|c|c|c|c|c|c|c|}
\hline \multirow[b]{2}{*}{ Study or Subgroup } & \multicolumn{2}{|c|}{ Experimental } & \multicolumn{4}{|c|}{ Control } & \multicolumn{2}{|r|}{ Mean Difference } & \multirow{2}{*}{\multicolumn{3}{|c|}{$\begin{array}{c}\text { Mean Difference } \\
\text { IV, Random, } 95 \% \mathrm{Cl}[\mathrm{bpm}]\end{array}$}} \\
\hline & Mean [bpm] & SD [bpm] & Total & Mean [bpm] & SD [bpm] & Total & Weight & IV, Random, 95\% Cl [bpm] & & & \\
\hline \multicolumn{12}{|c|}{ 5.2.1 Exenatide $10 \mathrm{mcg}$ vs active control } \\
\hline Barnett, 2007 & -1.29 & 8.7 & 68 & -0.93 & 8.95 & 70 & $5.3 \%$ & $-0.36[-3.30,2.58]$ & & & \\
\hline Bunck, 2009 & 4.6 & 10.02 & 36 & -2.2 & 9.82 & 33 & $2.5 \%$ & $6.80[2.12,11.48]$ & & & \\
\hline Buse, 2011 & 2.3 & 17.25 & 137 & -0.7 & 7.14 & 122 & $4.7 \%$ & $3.00[-0.15,6.15]$ & & & \\
\hline DeFronzo, 2010 & -0.6 & 9.34 & 73 & -1.67 & 10.03 & 79 & $4.9 \%$ & $1.07[-2.01,4.15]$ & & & \\
\hline Gallwitz, 2011 & -0.5 & 11 & 181 & 0.1 & 9.1 & 173 & $8.3 \%$ & $-0.60[-2.70,1.50]$ & & & \\
\hline Gallwitz, 2012 & 1.2 & 10.6 & 504 & 0.6 & 10.5 & 501 & $13.2 \%$ & $0.60[-0.70,1.90]$ & & & \\
\hline Heine, 2005 & -0.31 & 9.6 & 282 & -1.2 & 9.52 & 267 & $11.1 \%$ & $0.89[-0.71,2.49]$ & & & \\
\hline Nauck, 2007 & 1.53 & 10.27 & 253 & 1.19 & 9.17 & 248 & $10.5 \%$ & $0.34[-1.36,2.04]$ & & & \\
\hline Subtotal $(95 \% \mathrm{Cl})$ & & & 1534 & & & 1493 & $60.5 \%$ & $0.82[-0.15,1.79]$ & & & \\
\hline \multicolumn{12}{|c|}{ Heterogeneity: $\mathrm{Tau}^{2}=0.64 ; \mathrm{Chi}^{2}=10.83, \mathrm{df}=7(\mathrm{P}=0.15) ; \mathrm{I}^{2}=35 \%$} \\
\hline \multicolumn{12}{|c|}{ Test for overall effect: $Z=1.66(P=0.10)$} \\
\hline \multicolumn{12}{|c|}{ 5.2.2 Exenatide LAR $2.0 \mathrm{mg}$ vs active control } \\
\hline Bergenstal, 2010 (Piogl) & 1.6 & 9.27 & 80 & -0.9 & 8.75 & 165 & $6.9 \%$ & $2.50[0.07,4.93]$ & & & \\
\hline Bergenstal, 2010 (sita) & 1.6 & 9.27 & 80 & 0.5 & 8.93 & 166 & $6.9 \%$ & $1.10[-1.34,3.54]$ & & & \\
\hline Diamant, 2010 & 4 & 14.46 & 233 & 0 & 14.46 & 233 & $6.2 \%$ & $4.00[1.37,6.63]$ & & & \\
\hline Russell-Jones,2012 (MET) & 1.5 & 10 & 82 & 0.3 & 9.5 & 246 & $6.8 \%$ & $1.20[-1.27,3.67]$ & & & \\
\hline Russell-Jones,2012 (Sita) & 1.5 & 10 & 82 & 0.5 & 9.7 & 163 & $6.2 \%$ & $1.00[-1.63,3.63]$ & & & \\
\hline Russell-Jones,2012(Piogl) & 1.5 & 10 & 83 & -1.7 & 8.7 & 163 & $6.5 \%$ & $3.20[0.67,5.73]$ & & & \\
\hline Subtotal $(95 \% \mathrm{Cl})$ & & & 640 & & & 1136 & $39.5 \%$ & $2.14[1.11,3.17]$ & & & \\
\hline \multicolumn{12}{|c|}{ Heterogeneity: $\mathrm{Tau}^{2}=0.00 ; \mathrm{Chi}^{2}=4.66, \mathrm{df}=5(\mathrm{P}=0.46) ; \mathrm{l}^{2}=0 \%$} \\
\hline \multicolumn{12}{|c|}{ Test for overall effect: $Z=4.09(P<0.0001)$} \\
\hline Total $(95 \% \mathrm{Cl})$ & & & 2174 & & & 2629 & $100.0 \%$ & $1.36[0.57,2.14]$ & & & \\
\hline \multicolumn{9}{|c|}{ Heterogeneity: $\mathrm{Tau}^{2}=0.76 ; \mathrm{Chi}^{2}=20.48, \mathrm{df}=13(\mathrm{P}=0.08) ;\left.\right|^{2}=37 \%$} & & 1 & \\
\hline \multicolumn{9}{|c|}{ Test for overall effect: $Z=3.40(P=0.0007)$} & \multirow{2}{*}{\multicolumn{3}{|c|}{ Active control Exenatide }} \\
\hline Test for subgroup differen & $C h i^{2}=3.36, d$ & & & & & & & & & & \\
\hline
\end{tabular}

Figure 3 Effect of exenatide on heart rate in patients with type 2 diabetes. 
oral anti-diabetic drug (OAD) or insulin, were included. These doses were chosen to coincide with those most commonly used in clinical practice.

D. Comparison groups(s): Comparators included placebo, OAD, lifestyle intervention or insulin.

E. Outcomes: We included all studies reporting heart rate, blood pressure or body weight outcomes.

\section{Data extraction}

Retrieved studies were assessed for inclusion by two researchers independently using the above criteria, and any discrepancies were resolved by consensus. Information on the participants, intervention, comparison group, outcomes and trial quality was extracted from included studies by two researchers independently.

(a) GLP-1 vs placebo

Experimental

Control

Mean Difference

Mean Difference

Study or Subgroup Mean [mmHg] SD [mmHg] Total Mean [mmHg] SD [mmHg] Total Weight IV, Random, $95 \% \mathrm{Cl}[\mathrm{mmHg}] \quad$ IV, Random, $95 \% \mathrm{Cl}[\mathrm{mmHg}]$ 2.1.1 Liraglutide $1.2 \mathrm{mg}$ vs placebo

$\begin{array}{lrrr}\text { Nauck } 2009 & -2.8 & 14 & 242 \\ \text { Zinman } 2009 & -6.7 & 14.68 & 177 \\ \text { Subtotal }(95 \% \mathrm{Cl}) & & & 419 \\ \text { Heterogeneity: } \text { Tau }^{2}=7.08 ; \mathrm{Chi}^{2}=3.02, \mathrm{df}=1 & (\mathrm{P}=0.08) ; I^{2}=67 \% \\ \text { Test for overall effect: } \mathrm{Z}=1.38(\mathrm{P}=0.17) & & \\ \text { 2.1.2 Liraglutide 1.8 mg vs Placebo } & & \\ \text { Nauck 2009 } & -2.3 & 14 & 242 \\ \text { Russell- Jones, 2009 } & -4 & 15 & 230 \\ \text { Zinman 2009 } & -5.6 & 14.7 & 178 \\ \text { Subtotal }(95 \% \mathrm{Cl}) & & & 650\end{array}$

$\begin{array}{llll}-1.8 & 16 & 121 & 5.6 \%\end{array}$

$-1.1-15.96 \quad 89-4.7 \%$

$210 \quad 10.4 \%$

Heterogeneity: $\mathrm{Tau}^{2}=0.51 ; \mathrm{Chi}^{2}=2.32, \mathrm{df}=2(P=0.31) ; \mathrm{I}^{2}=14 \%$

Test for overall effect: $Z=2.13(P=0.03)$

2.1.3 Exenatide $5 \mathrm{mcg}$ vs placebo

DeFronzo, 2005

Kadowaki, 2009

Kendall 2005

Moretto, 2008

Subtotal $(95 \% \mathrm{Cl})$

$-2.24$

1.04

$15.34 \quad 125$

$14.29 \quad 110$

\begin{tabular}{ll}
$11.8 \quad 37$ \\
\hline
\end{tabular}

$13.92 \quad 245$

$-0.42$

$-3.56$

$11.68 \quad 77$

68
594

Heterogeneity: $\mathrm{Tau}^{2}=0.99 ; \mathrm{Chi}^{2}=4.79, \mathrm{df}=4(\mathrm{P}=0.31) ; \mathrm{l}^{2}=17 \%$

Test for overall effect: $Z=0.84(P=0.40)$

2.1.4 Exenatide $10 \mathrm{mcg}$ vs placebo

$\begin{array}{lrrr}\text { Apovian, 2010 } & -9.44 & 13.72 & 96 \\ \text { Buse, 2004 } & 0.82 & 15.71 & 129 \\ \text { Buse, 2011 } & -2.7 & 14.04 & 137 \\ \text { DeFronzo, 2005 } & -1.29 & 14.26 & 113 \\ \text { Gao, 2009 } & 0.8 & 14.44 & 234 \\ \text { Gill, 2010 } & -0.81 & 13.61 & 26 \\ \text { Kadowaki, 2009 } & -2 & 13.3 & 37 \\ \text { Kendall 2005 } & -2 & 14.98 & 241 \\ \text { Liutkus, 2010 } & -0.6 & 21.07 & 111 \\ \text { Moretto, 2008 } & -3.7 & 13.32 & 78 \\ \text { Zinman, 2007 } & -2.17 & 9.07 & 121 \\ \text { Subtotal (95\% Cl) } & & & 1323\end{array}$

Heterogeneity: $\mathrm{Tau}^{2}=6.27 ; \mathrm{Chi}^{2}=27.57, \mathrm{df}=10(\mathrm{P}=0.002) ;\left.\right|^{2}=64 \%$

Test for overall effect: $Z=1.67(P=0.09)$

2.1.5 Exenatide LAR $0.8 \mathrm{mg}$ vs placebo

Kim, 2007

$-0.3$

Subtotal $(95 \% \mathrm{Cl})$

$10.83 \quad 15$

Heterogeneity: Not applicable

Test for overall effect: $Z=0.09(P=0.93)$

2.1.6 Exenatide LAR $2.0 \mathrm{mg}$ vs placebo

Kim, 2007

$-4.1$

Subtotal $(95 \% \mathrm{Cl})$

$16.21 \quad 1$

Heterogeneity: Not applicable

Test for overall effect: $Z=0.66(P=0.51)$

Total (95\% Cl)

3017

Heterogeneity: $\mathrm{Tau}^{2}=3.19 ; \mathrm{Chi}^{2}=39.65, \mathrm{df}=22(\mathrm{P}=0.01) ; \mathrm{l}^{2}=45 \%$

Test for overall effect: $Z=3.05(P=0.002)$

Test for subaroup differences: $\mathrm{Chi}^{2}=1.49, \mathrm{df}=5(\mathrm{P}=0.91), \mathrm{l}^{2}=0 \%$

Figure 4 (Continued) 
Where necessary, clarification of data was obtained by correspondence with trial co-ordinators.

\section{Risk of bias}

We used the Cochrane tool to determine risk of selection bias (success of sequence generation and allocation concealment); performance bias (success of blinding to treatment received); detection bias (blinding of outcome assessment), attrition bias (incomplete outcome data and selective outcome reporting) and other biases. ${ }^{23}$ Funnel plots were used to detect publication bias.

\section{Analysis}

Means and SDs for baseline and outcome values for blood pressure, heart rate and body weight were extracted. Mean effect data from crossover trials were extracted at the end of the initial phase. Where SDs for the outcome were not available, they were imputed according to the Cochrane
Handbook for Systematic reviews V.5. ${ }^{23}$ SDs for changes from baseline were derived where necessary to account for correlation of baseline to follow-up measurements within individuals, and where the correlation coefficient could not be calculated, methods were employed as recommended by Follman et al. ${ }^{24}$ Study results were combined using RevMan V.5.2. Heterogeneity was estimated using the $\chi^{2}$ test and $\mathrm{I}^{2}$ statistic. Fixed and random effects weighted mean difference models using the Inverse Variance technique were used to compare outcomes between the study drug and comparator with $95 \%$ CI. Interaction effects were evaluated using prespecified subgroup analyses (comparing various doses of the study drug to active control or placebo) and type of GLP-1 agonist (liraglutide, exenatide twice daily and exenatide LAR preparations). Results are described using the random effects approach due to the heterogeneity of the included studies. Analyses were stratified by active control or placebo. We compared

(b) GLP-1 vs active control

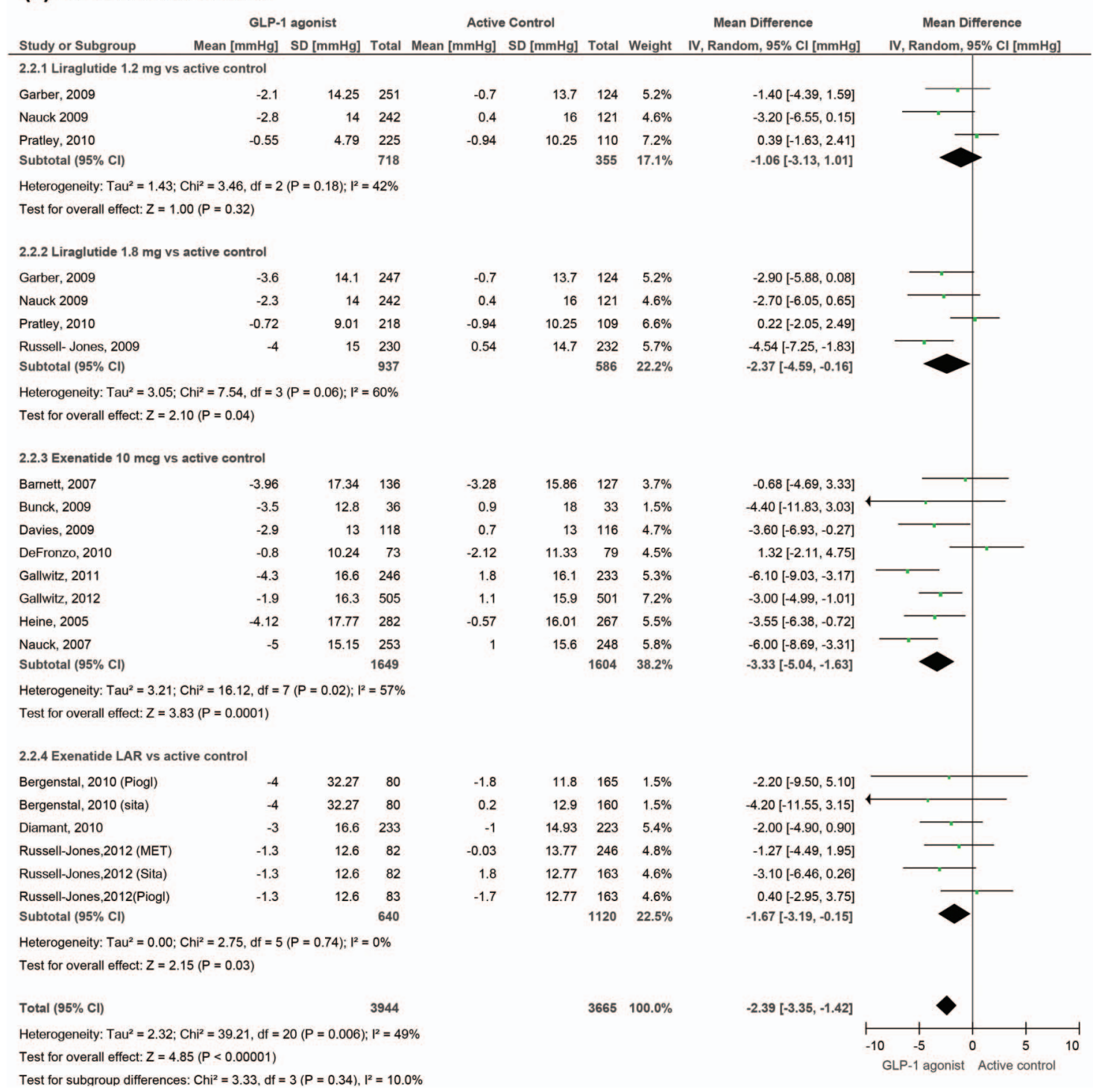

Figure 4 GLP-1 agonists' effect on systolic blood pressure in patients with type 2 diabetes. GLP-1, glucagon-like peptide-1. 
(a) GLP-1 vs placebo

Experimental Control Mean Difference Mean Difference Study or Subgroup Mean [mmHg] SD [mmHg] Total Mean [mmHg] SD [mmHg] Total Weight IV, Random, $95 \% \mathrm{Cl}[\mathrm{mmHg}] \quad$ IV, Random, 95\% Cl [mmHg] 3.1.1 Liraglutide $1.2 \mathrm{mg}$ vs placebo

\begin{tabular}{|c|c|c|c|}
\hline Zinman 2009 & -2.3 & 8.66 & 178 \\
\hline Subtotal $(95 \% \mathrm{Cl})$ & & & 178 \\
\hline \multicolumn{4}{|c|}{ Heterogeneity: Not applicable } \\
\hline \multicolumn{4}{|c|}{ Test for overall effect: $Z=1.44(P=0.15)$} \\
\hline \multicolumn{4}{|c|}{ 3.1.2 Liraglutide $1.8 \mathrm{mg}$ vs Placebo } \\
\hline Zinman 2009 & -1.9 & 8.07 & 178 \\
\hline Subtotal $(95 \% \mathrm{Cl})$ & & & 178 \\
\hline \multicolumn{4}{|c|}{ Heterogeneity: Not applicable } \\
\hline \multicolumn{4}{|c|}{ Test for overall effect: $Z=1.08(P=0.28)$} \\
\hline \multicolumn{4}{|c|}{ 3.1.3 Exenatide $5 \mathrm{mcg}$ vs placebo } \\
\hline Buse, 2004 & -1.96 & 9.05 & 125 \\
\hline DeFronzo, 2005 & -1 & 7.4 & 37 \\
\hline Kadowaki, 2009 & -1 & 7.4 & 37 \\
\hline Kendall 2005 & -0.73 & 8.58 & 245 \\
\hline Moretto, 2008 & -0.8 & 5.69 & 77 \\
\hline Subtotal $(95 \% \mathrm{Cl})$ & & & 521 \\
\hline
\end{tabular}

Heterogeneity: $\mathrm{Tau}^{2}=0.00 ; \mathrm{Chi}^{2}=1.04, \mathrm{df}=4(\mathrm{P}=0.90) ; \mathrm{I}^{2}=0 \%$

Test for overall effect: $Z=0.76(P=0.45)$

3.1.4 Exenatide $10 \mathrm{mcg}$ vs placebo

$\begin{array}{lrrr}\text { Apovian, 2010 } & -2.22 & 9.8 & 96 \\ \text { Buse, 2004 } & -0.47 & 9.38 & 129 \\ \text { Buse, 2011 } & -1.7 & 14.08 & 137 \\ \text { DeFronzo, 2005 } & -1 & 7.7 & 37 \\ \text { Gao, 2009 } & 0.7 & 8.55 & 234 \\ \text { Gill, 2010 } & -0.6 & 6.99 & 26 \\ \text { Kadowaki, 2009 } & -1 & 7.7 & 37 \\ \text { Kendall 2005 } & -0.41 & 8.9 & 241 \\ \text { Liutkus, 2010 } & -2.9 & 10.78 & 111 \\ \text { Moretto, 2008 } & -2.3 & 5.77 & 78 \\ \text { Zinman, 2007 } & -2.4 & 14.41 & 121 \\ \text { Subtotal (95\% Cl) } & & & 1247\end{array}$

$\begin{array}{rrrr}0.47 & 9.8 & 98 & 4.6 \% \\ -0.63 & 8.62 & 123 & 6.9 \% \\ 1.7 & 14.37 & 122 & 3.0 \% \\ -1 & 8 & 20 & 2.0 \% \\ -0.5 & 8.9 & 230 & 12.5 \% \\ -2.34 & 7.29 & 25 & 2.3 \% \\ -1 & 8 & 20 & 2.0 \% \\ -0.85 & 9 & 123 & 8.7 \% \\ -3.6 & 9.19 & 54 & 3.5 \% \\ -0.3 & 5.81 & 39 & 6.9 \% \\ 1.01 & 14.14 & 112 & 2.7 \% \\ & & 966 & 55.0 \%\end{array}$

Heterogeneity: $\mathrm{Tau}^{2}=1.21 ; \mathrm{Chi}^{2}=16.55, \mathrm{df}=10(\mathrm{P}=0.08) ; \mathrm{I}^{2}=40 \%$

Test for overall effect: $Z=0.94(P=0.35)$

3.1.5 Exenatide LAR $0.8 \mathrm{mg}$ vs placebo

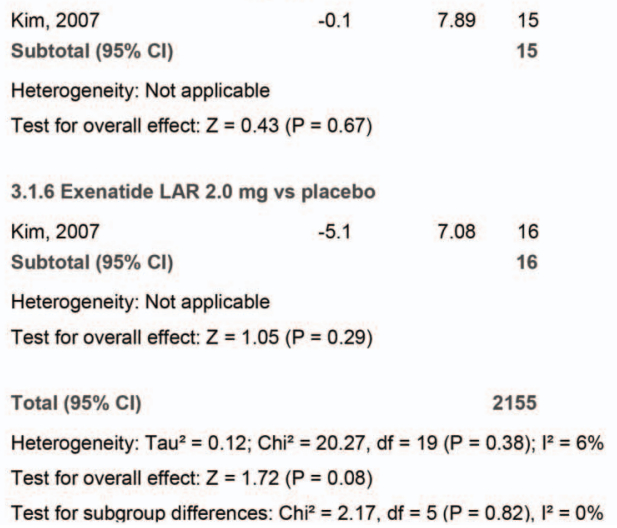

$-1.33[-3.53,0.87]$ $0.00[-4.24,4.24]$ $0.00[-4.24,4.24]$ $0.12[-1.79,2.03]$ $-0.50[-2.72,1.72]$ $-0.43[-1.56,0.69]$

$-2.69[-5.45,0.07]$ $0.16[-2.06,2.38]$ $-3.40[-6.87,0.07]$ $0.00[-4.30,4.30]$ $1.20[-0.39,2.79]$ $1.74[-2.18,5.66]$ $0.00[-4.30,4.30]$ $0.44[-1.51,2.39]$ $0.70[-2.47,3.87]$ $-2.00[-4.23,0.23]$ $-3.41[-7.08,0.26]$ $-0.52[-1.59,0.56]$

$-1.10[-3.10,0.90]$

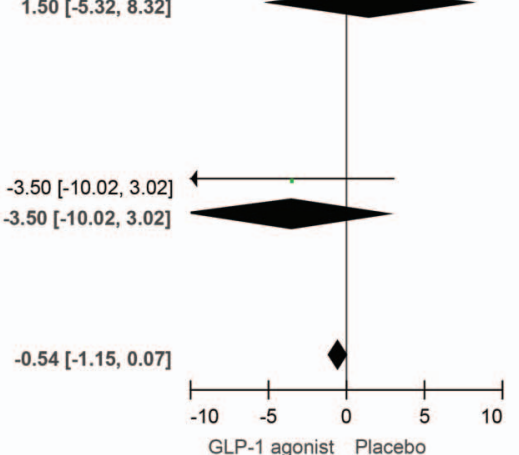

$1483 \quad 100.0 \%$ heterogeneity measures between these subgroups and according to the GLP-1 agent. We also undertook sensitivity analyses to investigate the influence of trial designs on heterogeneity measures, including the background OAD treatment common to both arms. Funnel plots were assessed for asymmetry.

\section{RESULTS}

Figure 1 describes the identification of the studies included. A total of 521 articles were screened. Of these, 472 were excluded on the basis of the title or abstract being irrelevant to the aims of this review. Forty-nine studies were examined in full text. Of these, four were 
(b) GLP-1 vs active control

\begin{tabular}{|c|c|c|c|c|c|c|c|c|c|}
\hline \multirow[b]{2}{*}{ Study or Subgroup } & \multicolumn{2}{|c|}{ Experimental } & \multicolumn{4}{|c|}{ Control } & \multicolumn{2}{|r|}{ Mean Difference } & \multirow{2}{*}{$\begin{array}{c}\text { Mean Difference } \\
\text { IV, Random, } 95 \% \mathrm{Cl}[\mathrm{mmHg}]\end{array}$} \\
\hline & Mean [mmHg] & $\mathrm{SD}[\mathrm{mmHg}]$ & Total & Mean $[\mathrm{mmHg}]$ & $\mathrm{SD}[\mathrm{mmHg}]$ & Total & Weight & IV, Random, $95 \% \mathrm{Cl}[\mathrm{mmHg}]$ & \\
\hline \multicolumn{9}{|c|}{ 3.2.1 Liraglutide $1.2 \mathrm{mg}$ vs active control } & \\
\hline Pratley, 2010 & -0.71 & 5.43 & 225 & -1.78 & 8.83 & 110 & $6.5 \%$ & $1.07[-0.73,2.87]$ & \\
\hline Subtotal $(95 \% \mathrm{Cl})$ & & & 225 & & & 110 & $6.5 \%$ & $1.07[-0.73,2.87]$ & \\
\hline \multicolumn{10}{|l|}{ Heterogeneity: Not applicable } \\
\hline \multicolumn{10}{|c|}{ Test for overall effect: $Z=1.17(P=0.24)$} \\
\hline \multicolumn{10}{|c|}{ 3.2.2 Liraglutide $1.8 \mathrm{mg}$ vs active control } \\
\hline Pratley, 2010 & 0.07 & 8.84 & 221 & -1.78 & 8.83 & 109 & $5.9 \%$ & $1.85[-0.18,3.88]$ & \\
\hline Subtotal $(95 \% \mathrm{Cl})$ & & & 221 & & & 109 & $5.9 \%$ & $1.85[-0.18,3.88]$ & \\
\hline \multicolumn{10}{|l|}{ Heterogeneity: Not applicable } \\
\hline \multicolumn{10}{|c|}{ Test for overall effect: $Z=1.79(P=0.07)$} \\
\hline \multicolumn{10}{|c|}{ 3.2.3 Exenatide $10 \mathrm{mcg}$ vs active control } \\
\hline Barnett, 2007 & -2 & 9.17 & 136 & -0.93 & 9.23 & 127 & $5.4 \%$ & $-1.07[-3.30,1.16]$ & \\
\hline Bunck, 2009 & -0.3 & 1.4 & 30 & 1.7 & 1.53 & 33 & $9.6 \%$ & $-2.00[-2.72,-1.28]$ & Tr \\
\hline Davies, 2009 & -0.5 & 7.6 & 118 & 0.9 & 7.14 & 117 & $6.3 \%$ & $-1.40[-3.29,0.49]$ & \\
\hline DeFronzo, 2010 & -1.17 & 9.48 & 73 & -2.24 & 8.65 & 79 & $4.1 \%$ & $1.07[-1.82,3.96]$ & \\
\hline Gallwitz, 2011 & -1.7 & 9.9 & 246 & 0.5 & 8.8 & 233 & $6.9 \%$ & $-2.20[-3.88,-0.52]$ & \\
\hline Gallwitz, 2012 & -1.3 & 10 & 505 & 0.3 & 10.3 & 501 & $8.1 \%$ & $-1.60[-2.85,-0.35]$ & \\
\hline Heine, 2005 & -1.18 & 10.33 & 282 & -0.77 & 9.76 & 267 & $6.9 \%$ & $-0.41[-2.09,1.27]$ & \\
\hline Nauck, 2007 & -1.8 & 9.68 & 253 & 0.6 & 10.13 & 248 & $6.7 \%$ & $-2.40[-4.14,-0.66]$ & \\
\hline Subtotal $(95 \% \mathrm{Cl})$ & & & 1643 & & & 1605 & $54.0 \%$ & $-1.63[-2.18,-1.08]$ & \\
\hline \multicolumn{10}{|c|}{ Heterogeneity: $\mathrm{Tau}^{2}=0.07 ; \mathrm{Chi}^{2}=7.84, \mathrm{df}=7(\mathrm{P}=0.35) ; \mathrm{I}^{2}=11 \%$} \\
\hline \multicolumn{10}{|c|}{ Test for overall effect: $Z=5.82(P<0.00001)$} \\
\hline \multicolumn{10}{|c|}{ 3.2.4 Exenatide LAR $2.0 \mathrm{mg}$ vs active control } \\
\hline Bergenstal, 2010 (Piogl) & -1.6 & 7.46 & 80 & -2.6 & 0.6 & 137 & $7.0 \%$ & $1.00[-0.64,2.64]$ & \\
\hline Bergenstal, 2010 (sita) & -1.6 & 7.46 & 80 & -0.2 & 8.12 & 137 & $5.7 \%$ & $-1.40[-3.53,0.73]$ & \\
\hline Diamant, 2010 & -1 & 14.46 & 233 & -1 & 14.46 & 223 & $4.5 \%$ & $0.00[-2.66,2.66]$ & \\
\hline Russell-Jones,2012 (MET) & -0.65 & 8.55 & 82 & -0.6 & 8.47 & 246 & $5.7 \%$ & $-0.05[-2.18,2.08]$ & \\
\hline Russell-Jones,2012 (Sita) & -0.65 & 8.55 & 82 & -0.51 & 7.76 & 162 & $5.5 \%$ & $-0.14[-2.34,2.06]$ & \\
\hline Russell-Jones,2012(Piogl) & -0.65 & 8.55 & 83 & -2.67 & 9.15 & 163 & $5.2 \%$ & $2.02[-0.29,4.33]$ & \\
\hline Subtotal $(95 \% \mathrm{Cl})$ & & & 640 & & & 1068 & $33.5 \%$ & $0.29[-0.63,1.20]$ & \\
\hline \multicolumn{10}{|c|}{ Heterogeneity: $\operatorname{Tau}^{2}=0.14 ; \mathrm{Chi}^{2}=5.58, \mathrm{df}=5(\mathrm{P}=0.35) ; \mathrm{I}^{2}=10 \%$} \\
\hline \multicolumn{10}{|c|}{ Test for overall effect: $Z=0.61(P=0.54)$} \\
\hline Total $(95 \% \mathrm{Cl})$ & & & 2729 & & & 2892 & $100.0 \%$ & $-0.50[-1.24,0.24]$ & \\
\hline \multicolumn{9}{|c|}{ Heterogeneity: $\mathrm{Tau}^{2}=1.35 ; \mathrm{Chi}^{2}=42.27, \mathrm{df}=15(\mathrm{P}=0.0002) ;\left.\right|^{2}=65 \%$} & -4 \\
\hline \multicolumn{9}{|c|}{ Test for overall effect: $Z=1.33(P=0.18)$} & 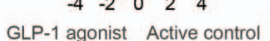 \\
\hline
\end{tabular}

Figure 5 GLP-1 agonists' effect on diastolic blood pressure in patients with type 2 diabetes. GLP-1, glucagon-like peptide-1.

excluded because the comparator was another form of GLP-1. ${ }^{25-28}$ In three cases, the doses were not as specified in our inclusion criteria, ${ }^{29-31}$ and in a further two, the study involved further analysis of data from trials that were already included. ${ }^{32}$ Finally, eight were open label extension studies. ${ }^{34-41}$ This left 32 trials included in our review (figure 1 and table 1). ${ }^{4-73}$ Most studies did not report all of the outcomes of interest, or they did not provide them as usable numerical data. Data were therefore obtained, where available, directly from the pharmaceutical companies.

\section{Methodological quality and risk of bias}

Results of risk of bias assessment are given in table 2. Explanation of sequence generation and allocation concealment was adequate for all trials. In nine trials, at least one arm was open label. Attrition was adequately described and was greater than $20 \%$ in nine studies. The proportion of the intention-to-treat population completing the study varied in range $65.4-99.6 \%$ and had a median of $83.7 \%$. None of the trials were terminated prematurely. Funnel plots were broadly symmetrical with no evidence of publication bias.

\section{Heterogeneity}

The trials varied in terms of duration of follow-up, location, type of active comparator drug and background therapy. One study was a crossover trial ${ }^{43}$ and another was of a prolonged follow-up. ${ }^{55}$ The mean age of the participants ranged from 52.3 to 60.3 years. For most outcomes, we found significant heterogeneity (figures 2-6). We, therefore, chose to report results using the random effects approach, although the differences between random effects and fixed effect results were very small. Heterogeneity varied significantly between comparisons. For the effect of liraglutide on heart rate compared with placebo, the $\mathrm{I}^{2}$ value was $55 \%$. 
However, this value reduced to $0 \%$ when the data from a single trial (LEAD-1) were withheld.

\section{Heart rate}

A total of 22 studies provided heart rate data. Overall, GLP-1 agonists produce a significant increase in heart rate with a weighted mean difference of $1.86 \mathrm{bpm}$ (0.85 to 2.87) versus placebo and $1.90 \mathrm{bpm}$ (1.30 to $2.50)$ versus active control. Looking at specific agents, liraglutide increases heart rate by $2.71 \mathrm{bpm}$ (1.45 to 3.97 ) versus placebo and 2.49 (1.77 to 3.21 ) versus active control. Data from the LEAD trials of liraglutide 5764676973 were initially grouped into quartiles of baseline heart rate and demonstrated significant variations in effect between these subgroups, with the greatest increase seen in those with the lowest baseline values. Exenatide twice daily increased the heart rate by $0.82 \mathrm{bpm}(-0.15$ to 1.79$)$ versus active control and by $0.88 \mathrm{bpm}$ ( -0.47 to 2.22$)$ versus placebo, which did not reach statistical significance (figure 3). Exenatide LAR produced a more significant change $(2.14 \mathrm{bpm}$ (1.11 to 3.17 ) versus active control), but the number of studies involving this formulation was small.

\section{Blood pressure}

We included 31 trials measuring blood pressure changes (figures 4 and 5). GLP-1 agonists reduced systolic blood pressure by $-1.79 \mathrm{~mm} \mathrm{Hg}(-2.94$ to -0.64$)$ compared to placebo and by -2.39 ( -3.35 to -1.42$)$ compared to active control. Reductions in diastolic blood pressure failed to reach statistical significance and were $-0.54 \mathrm{~mm} \mathrm{Hg}(-1.15$ to 0.07$)$ compared to placebo and $-0.50 \mathrm{~mm} \mathrm{Hg}(-1.24$ to 0.24) compared to active control.

\section{Body weight}

Twenty-one trials measuring changes in weight were included (figure 6). We confirm a small but highly significant reduction in body weight as a result of GLP-1 therapy. Weight changed by $-3.31 \mathrm{~kg}(-4.05$ to -2.57$)$

\section{(a) GLP-1 agonists versus placebo}

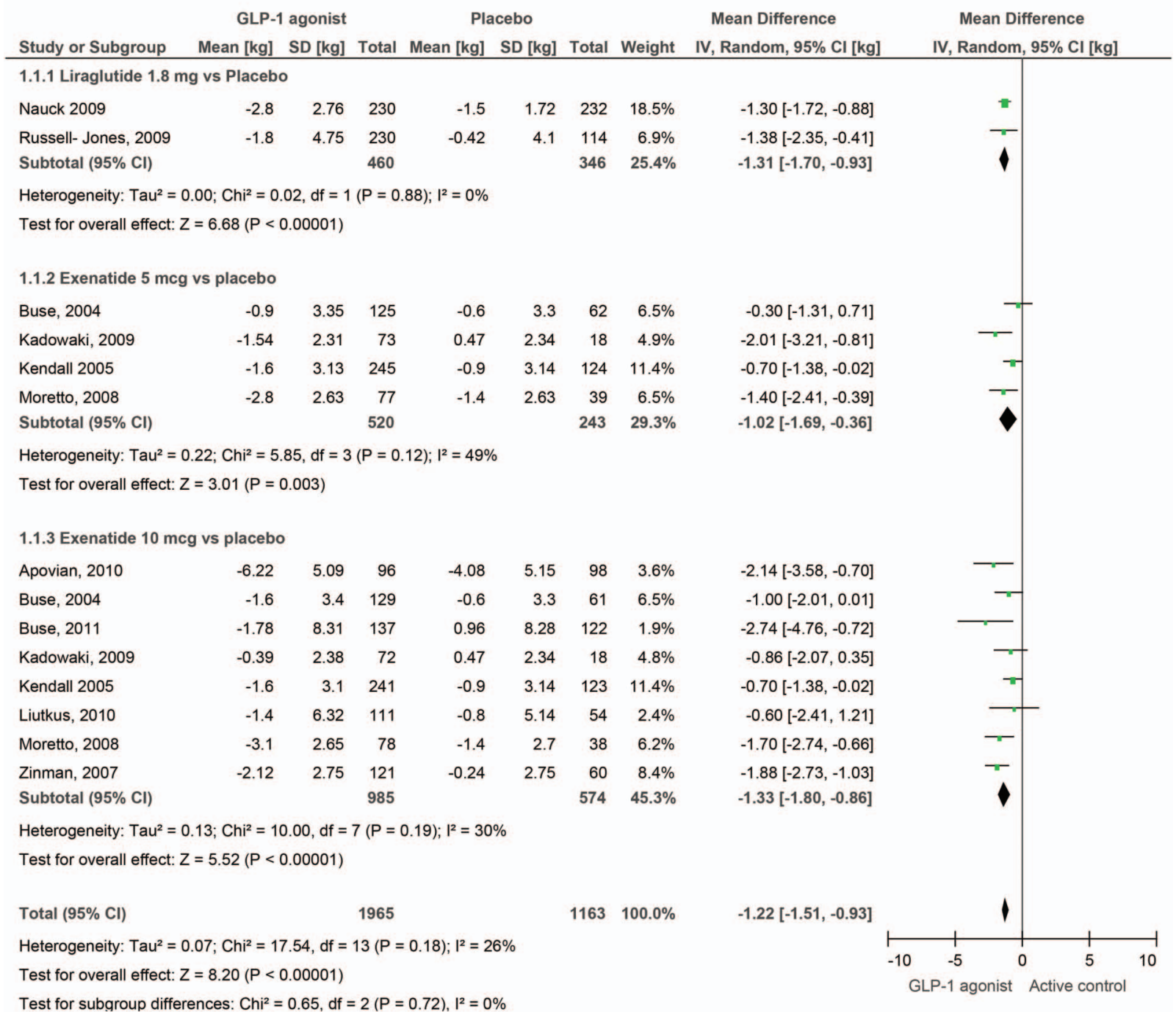

Figure 6 (Continued) 
compared to active control but by only $-1.22 \mathrm{~kg}$ $(-1.51$ to -0.93$)$ compared to placebo.

\section{DISCUSSION}

We have confirmed and quantified the effects of liragutide and exenatide on heart rate, blood pressure and body weight. Our analysis benefited from the inclusion of unpublished data supplied by Novo Nordisk and Amylin Pharmaceuticals, as these were often missing from published trial reports. It was limited by the significant heterogeneity of effect size measurements between individual studies. We examined prespecified subgroups according to the GLP-1 agent and type of comparator (placebo or active control). Active control treatments varied between trials and included different classes of OAD and insulins, which may explain some of the variation in measured effect. Other potential sources of heterogeneity include the characteristics of background OAD treatments common to both arms as these treatments differed between trials. For the heart rate effect of liraglutide versus placebo, the heterogeneity was largely attributable to a single trial (LEAD-1), but the cause of the higher heart rate effect in this trial is unclear.

The weight-reducing effects of these agents are a welcome contrast to the weight-promoting effects of other treatment options, including sulphonylureas, thiazolidinediones and insulin. We have derived a similar

\section{(b) GLP-1 agonists versus active control}

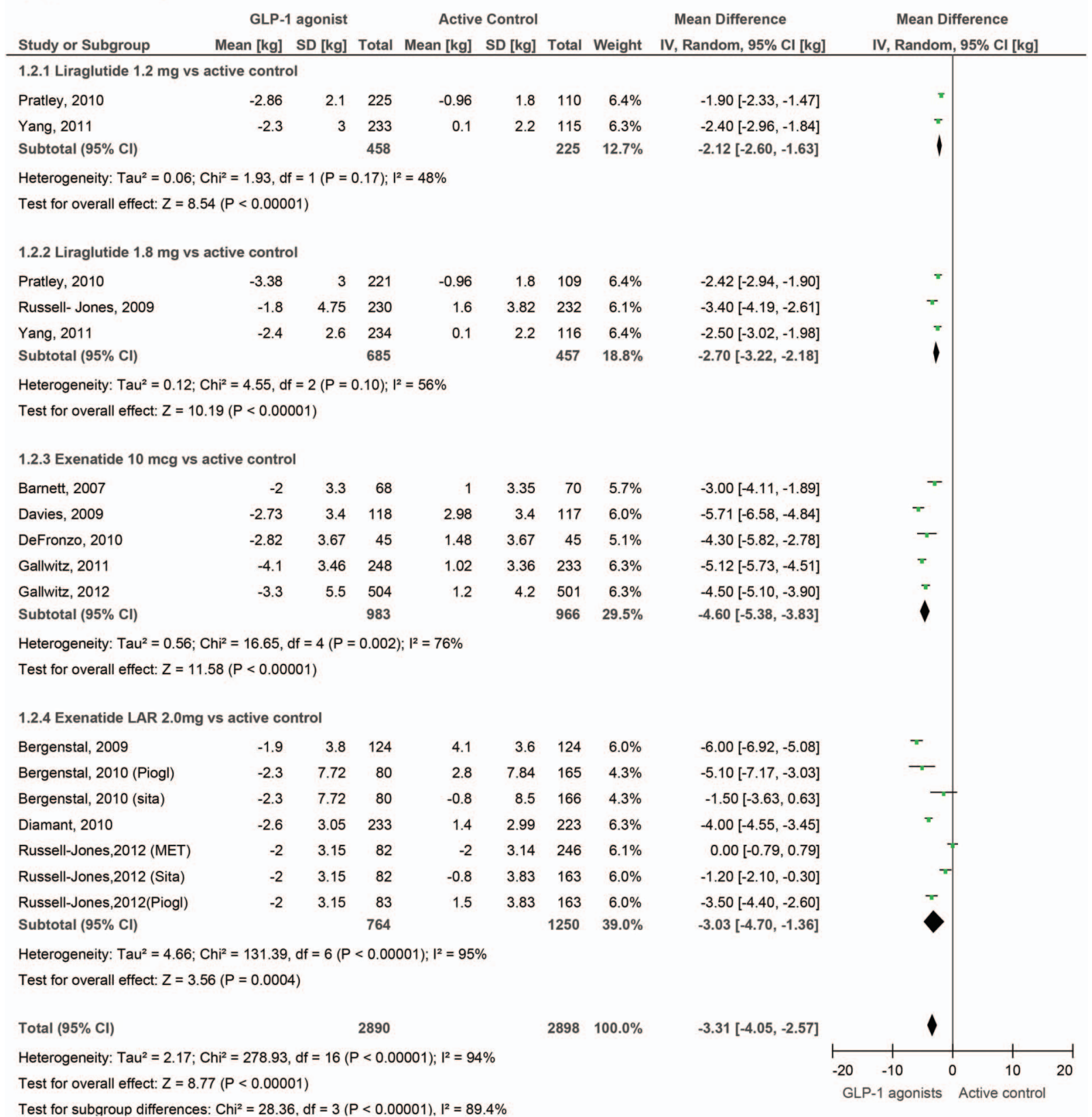

Figure 6 GLP-1 agonists' effects on body weight. GLP-1, glucagon-like peptide-1. 
effect size to a previously reported value for weight loss, ${ }^{2}$ although our study has distinguished between placebo and active comparators, in which effects sizes differ substantially. Together with the reduction in blood pressure, this may improve longer term cardiovascular risk. However, the small rise in heart rate is a reason for caution, as it might potentially be associated with adverse outcomes. This rise was more evident for liraglutide than exenatide twice daily, but exenatide LAR may produce a greater response than the twice-daily formulation. The clinical significance of this heart rate rise is still unknown from the perspective of cardiovascular risk.

For most GLP-1 trials, heart rate is a secondary outcome measured as part of safety assessment, and is reported inconsistently. In clinic, it is often measured using a very short sampling interval (perhaps $1 \mathrm{~min}$ of data). One study was designed specifically to examine the effects of exenatide twice daily on change in heart rate as the primary outcome using $24 \mathrm{~h}$ ambulatory monitoring. ${ }^{58}$ The mean change from baseline at 12 weeks was $2.1 \mathrm{bpm}$ for exenatide twice daily and $-0.7 \mathrm{bpm}$ for placebo. The sample size (54 randomised participants) in this pilot study was relatively small and the difference was not significant $(p=0.16)$, but it is similar to the values we have obtained generally for GLP-1 agonists in our meta-analysis. Measurement of heart rate using this $24 \mathrm{~h}$ technique (compared with a traditional heart rate measurement in clinic) substantially improves the accuracy of measurement as heart rate is very variable within the individual. This technique could be used as a basis for a larger study powered to detect such a difference and to investigate the influence of alternative background medications.

This review highlights the need to improve our understanding of the physiological mechanisms through which GLP-1 agonists act, while the results of longer term safety studies are awaited. Both autonomic nervous system-dependent and system-independent effects have been suggested in animal studies as a basis for the rise in heart rate. ${ }^{74}$ The heart rate response in the presence or absence of autonomic neuropathy in human patients might therefore justify further study. There is also a clear need to improve the comprehensive reporting of all outcome data measured during clinical trials of antidiabetic agents, particularly those relevant to cardiovascular risk.

Acknowledgements We would like to thank Amylin Pharmaceuticals and Novo Nordisk for providing us with unpublished data for this meta-analysis.

Contributors LER, JPO and HSR were involved in the design and conception of the study. LER and TAH conducted the bibliographic searches, identified the included papers and extracted the data independently. KR advised on methodological issues. All authors were involved in the drafting of the manuscript.

Funding This research received no specific grant from any funding agency in the public, commercial or not-for-profit sectors.

Competing interests LER, TAH and KR have no interests to declare. JPO and HSR have received research funding (paid to Warwick Medical School) from Novo Nordisk, and payments for speaking from Novo Nordisk.
Data sharing statement All data used in this study are freely available by request to the corresponding author TAH.

\section{REFERENCES}

1. Nathan DM, Buse JB, Davidson MB, et al. Medical management of hyperglycemia in type 2 diabetes: a consensus algorithm for the initiation and adjustment of therapy. Diabetes Care 2009;32:193-203.

2. Vilsboll $T$, Christensen $M$, Junker $A E$, et al. Effects of glucagon-like peptide receptor agonists on weight loss: systematic review and meta-analyse of randomized controlled trials. BMJ 2012;344:1-11.

3. Flint A, Raben A, Astrup A, et al. Glucagon-like peptide 1 promotes satiety and suppresses energy intake in humans. J Clin Invest 1998;101:515-20.

4. MacDonald PE, El-kholy W, Riedel MJ, et al. The multiple actions of GLP-1 on the process of glucose-stimulated insulin secretion. Diabetes 2002;51(Suppl 3):S434-42.

5. Willms B, Werner J, Holst JJ, et al. Gastric emptying, glucose responses, and insulin secretion after a liquid test meal: effects of exogenous glucagon-like peptide-1 (GLP-1)-(7-36) amide in type 2 (noninsulin-dependent) diabetic patients. J Clin Endocrinol Metab 1996;81:327-32.

6. Meier JJ, Nauck MA. Glucagon-like peptide 1(GLP-1) in biology and pathology. Diabetes Metab Res Rev 2005;21:91-117.

7. Varanasi A, Chaudhuri A, Dhindsa S, et al. Durability of effects of exenatide on glycaemic control, body weight, systolic blood pressure, C-reactive protein and triglyceride concentrations. Endocrin Prac 2011;17:192-200.

8. Bojanowska $E$, Stempniak B. Effects of glucagon-like peptide-1 (7-36) amide on neurohypophysial hormone secretion induced by acute hyperosmotic challenge. Neuropeptides 2003;37:45-50.

9. Bharucha AE, Charkoudian N, Andrews CN, et al. Effects of glucagon-like peptide-1, yohimbine, and nitrergic modulation on sympathetic and parasympathetic activity in humans. Am J Physiol Regul Integr Comp Physiol 2008;295:R874-80.

10. Levine HJ. Rest heart rate and life expectancy. J Am Coll Cardiol 1997;30:1104-6.

11. Aronow WS, Ahn C, Mercando AD, et al. Association of average heart rate on 24-hour ambulatory electrocardiograms with incidence of new coronary events at 48-month follow-up in 1311 patients (mean age 81 years) with heart disease and sinus rhythm. Am J Cardiol 1996;78:1175-6.

12. Hozawa A, Ohkubo T, Kikuya M, et al. Prognostic value of home heart rate for cardiovascular mortality in the general population: the Ohasama study. Am J Hypertens 2004;17:1005-10.

13. Anselmino M, Öhrvik J, Rydén L. Resting heart rate in patients with stable coronary artery disease and diabetes: a report from the euro heart survey on diabetes and the heart. Eur Heart $J$ 2010;31:3040-5.

14. Bode B. An overview of the pharmacokinetics, efficacy and safety of liraglutide. Diab Res Clin Pract 2012;97:27-42.

15. Fakhoury WKH, Lereun C, Wright D. A meta-analysis of placebo-controlled clinical trials assessing the efficacy and safety of incretin-based medications in patients with type 2 diabetes. Pharmacol 2010;86:44-57.

16. Blonde L, Russell-Jones D. The safety and efficacy of liraglutide with or without oral antidiabetic drug therapy in type 2 diabetes: an overview of the LEAD 1-5 studies. Diabetes Obes Metab 2009;11 (Suppl 3):26-34.

17. Ryder REJ, Thong KY, Cull ML, et al. on behalf of the ABCD nationwide exenatide audit contributors. The Association of British Clinical Diabetologists (ABCD) nationwide exenatide audit. Practical Diabetes Int 2010;27:352-7.

18. http://www.diabetologists-abcd.org.uk/Objectives_Liraglutide_Audit. htm (accessed 18 Apr 2012).

19. National Institute for Health and Clinical Excellence. Technology Appraisal TA248. Exenatide prolonged-release suspension for injection in combination with oral antidiabetic therapy for the treatment of type 2 diabetes. London: NICE, 2012.

20. Madsbad S, Kielgast U, Asmar M, et al. An overview of once-weekly glucagon-like peptide-1 receptor agonists-available efficacy and safety data and perspectives for the future. Diabetes Obes Metab 2011;13:394-407.

21. Gustavson SM, Chen D, Somayaji V, et al. Effects of a long-acting GLP-1 mimetic (PF-04603629) on pulse rate and diastolic blood pressure in patients with type 2 diabetes mellitus. Diabetes Obes Metab 2011;13:1056-8.

22. Ratner R, Han J, Nicewarner D, et al. Cardiovascular safety of exenatide BID: an integrated analysis from controlled clinical trials in 
participants with type 2 diabetes. Cardiovasc Diabetol

2011;10:22-32.

23. Higgins JPT, Green S Cochrane handbook for systematic reviews of interventions. Version 5.1.0 (updated March 2011). The Cochrane Collaboration, 2011. http://www.cochrane-handbook.org (accessed 15 Nov 2012)

24. Follman D, Elliott $\mathrm{P}$, Suh I, et al. Variance imputation for overviews of clinical trials with continuous response. J Clin Epidemiol 1992;45:769-73.

25. Blevins T, Pullman J, Malloy J, et al. DURATION 5: exenatide once weekly resulted in greater improvements in glycemic control compared with exenatide twice daily in patients with type 2 diabetes. $J$ Clin Endocrinol Metab 2011;96:1301-10.

26. Buse JB, Rosenstock J, Sesti G, et al. Liraglutide once a day versus exenatide twice a day for type 2 diabetes: a 26-week randomised, parallel-group, multinational, open label trial (LEAD-6). Lancet 2009;374:39-47.

27. Buse JB, Drucker DJ, Taylor KL, et al. DURATION-1: exenatide once weekly produces sustained glycemic control and weight loss over 52 weeks. Diabetes Care 2010;33:1255-61.

28. Drucker D, Buse JB, Taylor K, et al. Exenatide once-weekly versus twice daily for the treatment of type 2 diabetes: a randomized open-label, non-inferiority study. Lancet 2008;372:1240-50.

29. Bunck MC, Diamant M, Corner A, et al. One-year treatment with exenatide improves beta-cell function, compared with insulin glargine, in metformin-treated type 2 diabetic patients: a randomized, controlled trial. Diabetes Care 2009;32:762-8.

30. Feinglos MN, Saad MF, Pi-Sunyer FX, et al. Effects of liraglutide (NN2211), a long-acting GLP-1 analogue, on glycaemic control and bodyweight in subjects with Type 2 diabetes. Diabet Med 2005:22:1016-23.

31. Vilsboll T, Zdravkovic M, Le-Thi T, et al. Liraglutide, a long-acting human glucagon-like peptide-1 analog, given as monotherapy significantly improves glycemic control and lowers body weigh without risk of hypoglycemia in patients with type 2 diabetes. Diabetes Care 2007;30:1608-10.

32. Bode BW, Testa MA, Magwire M, et al. Patient-reported outcomes following treatment with the human GLP-1 analogue liraglutide or glimepiride in monotherapy: results from a randomized controlled trial in patients with type 2 diabetes. Diabetes Obes Metab 2010;12:604-12.

33. Davies M, Pratley R, Hammer M, et al. Liraglutide improves treatment satisfaction in people with type 2 diabetes compared with sitagliptin, each as an add on to metformin. Diabet Med 2011;28:333-7.

34. Bunck MC, Cornér A, Eliasson $B$, et al. One year treatment with exenatide vs. insulin glargine: effects on post-prandial glycemia, lipid profiles and oxidative stress. Atherosclerosis 2010;212:223-9.

35. Bunck MC, Cornér A, Eliasson B, et al. Effects of exenatide on measures of beta-cell function after 3 years in metformin-treated patients with type 2 diabetes. Diabetes Care 2011;34:2041-7.

36. Buse JB, Klonoff DC, Nielsen LL, et al. Metabolic effects of two years of exenatide treatment on diabetes, obesity, and hepatic biomarkers in patients with type 2 diabetes: an interim analysis of data from the open-label, uncontrolled extension of three double-blind, placebo-controlled trials. Clin Ther 2007;29:139-53.

37. Klonoff DC, Buse JB, Nielsen LL, et al. Exenatide effects on diabetes, obesity, cardiovascular risk factors and hepatic biomarkers in patients with type 2 diabetes treated for at least 3 years. Curr Med Res Opin 2008;24:275-86.

38. Pratley R, Nauck M, Bailey $\mathrm{T}$, et al. One year of liraglutide treatment offers sustained and more effective glycaemic control and weight reduction compared with sitagliptin, both in combination with metformin, in patients with type 2 diabetes: a randomised, parallel-group, open-label trial. Int J Clin Pract 2011;65:397-407.

39. Ratner RE, Maggs D, Nielsen LL., et al Long term effects of exenatide therapy over 82 weeks on glycemic control and weight in overweight metformin treated patients with type 2 diabetes mellitus. Diabetes Obes Metab 2006;8:419-28.

40. Riddle MC, Henry RR, Poon TH, et al. Exenatide elicits sustained glycaemic control and progressive reduction of body weight in patients with type 2 diabetes inadequately controlled by sulphonylureas with or without metformin. Diabetes Metab Res Rev 2006;22:483-91.

41. Wysham C, Bergenstal R, Malloy J, et al. DURATION-2: efficacy and safety of switching from maximum daily sitagliptin or pioglitazone to once-weekly exenatide. Diabet Med 2011;28:705-14.

42. Apovian CM, Bergenstal RM, Cuddihy RM, et al. Effects of exenatide combined with lifestyle modification in patients with type 2 diabetes. Am J Med 2010;123:468-77.

43. Barnett AH, Burger J, Johns D, et al. Tolerability and efficacy of exenatide and titrated insulin glargine in adult patients with type 2 diabetes previously uncontrolled with metformin or a sulphonylurea: a multinational, randomised, open label two period crossover non-inferiority trial. Clin Ther 2007;29:2333-48.

44. Bergenstal R, Lewin A, Bailey $\mathrm{T}$, et al. Efficacy and safety of biphasic insulin aspart $70 / 30$ versus exenatide in subjects with type 2 diabetes failing to achieve glycemic control with metformin and a sulfonylurea. Curr Med Res Opin 2009;25:65-75.

45. Bergenstal RM, Wysham C, MacConell L, et al. Efficacy and safety of exenatide once weekly versus sitagliptin or pioglitazone as an adjunct to metformin for treatment of type 2 diabetes (DURATION-2): a randomised trial. Lancet 2010;376:431-9.

46. Buse JB, Henry RR, Han J, et al. Effects of exenatide (exendin-4) on glycaemic control over 30 weeks in patients with type 2 diabetes treated with metformin and a sulphonylurea. Diabetes Care 2004;27:2628-35

47. Buse JB, Bergenstal RM, Glass LC, et al. Use of twice daily exenatide in basal insulin-treated patients with type 2 diabetes. A randomized controlled trial. Ann Int Med 2011;154:103-12.

48. Davies MJ, Donnelly $\mathrm{R}$, Barnett $\mathrm{AH}$, et al. Exenatide compared with long-acting insulin to achieve glycaemic control with minimal weight gain in patients with type 2 diabetes: results of helping evaluate exenatide in patients with diabetes compared with long-acting insulin (HEELA) study. Diabetes Obes Metab 2009;11:1153-64.

49. DeFronzo RA, Ratner RE, Han J, et al. Effects of exenatide (exendin-4) on glycemic control and weight over 30 weeks in metformin-treated patients with type 2 diabetes. Diabetes Care 2005;28:1092-100.

50. Defronzo RA, Triplitt C, Qu Y, et al. Effects of exenatide plus rosiglitazone on beta-cell function and insulin sensitivity in subjects with type 2 diabetes on metformin. Diabetes Care 2010;33:951-7.

51. Derosa G, Maffioli P, Salvadeo SA, et al. Exenatide vs. glibenclamide in patients with diabetes. Diabetes Technol Therap 2010;12:233-40.

52. Derosa G, Putignano P, Bossi AC, et al. Exenatide or glimepiride added to metformin on metabolic control and on insulin resistance in type 2 diabetic patients. Eur J Pharmacol 2011;666:251-6.

53. Diamant M, Gaal L, Stranks S, et al. Once weekly exenatide compared with insulin glargine titrated to target in patients with type 2 diabetes (DURATION 3): An open-label randomised trial. Lancet 2010;375:2234-43.

54. Gallwitz B, Bohmer M, Segiet T, et al. Exenatide twice daily versus premixed insulin aspart 70/30 in metformin-treated patients with type 2 diabetes: a randomized 26-week study on glycemic control and hypoglycaemia. Diabetes Care 2011;34:604-6.

55. Gallwitz B, Guzman J, Dotta F, et al. Exenatide twice daily versus glimepiride for prevention of glycaemic deterioration in patients with type 2 diabetes with metformin failure (EUREXA): an open-label, randomised controlled trial. Lancet 2012;379:2270-8.

56. Gao Y, Yoon $\mathrm{KH}$, Chuang L-M, et al. Efficacy and safety of exenatide in patients of Asian descent with type 2 diabetes inadequately controlled with metformin or metformin and a sulphonylurea. Diabetes Res Clin Pract 2009;83:69-76.

57. Garber A, Henry R, Ratner R, et al., for the LEAD-3 (Mono) Study Group. Liraglutide versus glimepiride monotherapy for type 2 diabetes (LEAD-3 Mono): a randomised, 52-week, phase III, double-blind, parallel-treatment trial. Lancet 2009;373:473-81.

58. Gill A, Hoogwerf BJ, Burger J, et al. Effect of exenatide on heart rate and blood pressure in subjects with type 2 diabetes mellitus: a double blind, placebo controlled, randomized pilot study. Cardiovasc Diabetol 2010;9:6.

59. Heine RJ, Van Gaal LF, Johns D, et al., GWAA Study Group. Exenatide vs insulin glargine in patients with suboptimally controlled type 2 diabetes: a randomised trial. Ann Intern Med 2005:143:559-69.

60. Kadowaki T, Namba M, Yamamura A, et al. Exenatide exhibits dose-dependent effects on glycemic control over 12 weeks in Japanese patients with suboptimally controlled type 2 diabetes. Endocr J 2009;56:415-24.

61. Kendall DM, Riddle MC, Rosenstock J, et al. Effects of exenatide (Exendin-4) on glycaemic control over 30 weeks in patients with type 2 diabetes treated with metformin and a sulphonylurea. Diabetes Care 2005:28:1083-91.

62. Kim D, MacConell L, Zhuang D, et al. Effects of once-weekly dosing of a long-acting release formulation of exenatide on glucose control and body weight in subjects with type 2 diabetes. Diabetes Care 2007;30:1487-93.

63. Liutkus J, Guzman JR, Norwood P, et al. A placebo-controlled trial of exenatide twice-daily added to thiazolidinediones alone or in combination with metformin. Diabetes Obes Metab 2010;12:1058-65.

64. Marre M, Shaw J, Brandle M, et al. Liraglutide once-daily human GLP-1 analogue, added to a sulphonylurea over 26 weeks produces 
greater improvements in glycaemic and weight control compared with adding rosiglitazone or placebo in subjects with type 2 diabetes (Lead 1-SU). Diabet Med 2009;26:268-78.

65. Moretto TJ, Milton DR, Ridge TD., et al Efficacy and tolerability of exenatide monotherapy over 24 weeks in antidiabetic drug-naive patients with type 2 diabetes: a randomized, double blind, placebo-controlled parallel-group study. Clin Ther 2008;30:1448-60.

66. Nauck MA, Duran S, Kim D, et al. A comparison of twice-daily exenatide and biphasic insulin aspart in patients with type 2 diabetes who were suboptimally controlled with sulfonylurea and metformin: a non-inferiority study. Diabetologia 2007;50:259-67.

67. Nauck M, Frid A, Hermansen K, et al. Efficacy and safety comparison of liraglutide, glimepiride and placebo, all in combination with metformin in type 2 diabetes. Diabetes Care 2009;32:84-90.

68. Pratley RE, Nauck M, Bailey $T$, et al. Liraglutide vs sitagliptin for patients with type 2 diabetes who did not have adequate glycemic control with metformin: a 26 week, randomised, parallel group, open-label trial. Lancet 2010;375:1447-56.

69. Russell-Jones D, Vaag A, Schmitz O, et al. Liraglutide vs insulin glargine and placebo in combination with metformin and sulfonylurea therapy in type 2 diabetes mellitus (LEAD-5 met+SU): a randomised controlled trial. Diabetologia 2009;52:2046-55.

70. Russell-Jones D, Cuddihy RM, Hanefeld M, et al. Efficacy and safety of exenatide once weekly versus metformin, pioglitazone, and sitagliptin used as monotherapy in drug-naïve patients with type 2 diabetes (DURATION-4): a 26-week double-blind study. Diabetes Care 2012;35:252-8.

71. Yang W, Chen L, Ji Q, et al. Liraglutide provides similar glycaemic control as glimepiride (both in combination with metformin) and reduces body weight and systolic blood pressure in Asian population with type 2 diabetes from China, South Korea and India: a 16-week, randomized, double-blind, active control trial. Diabetes Obes Metab 2011;13:81-8

72. Zinman B, Hoogerworf BJ, Garcia SD, et al. The effect of adding exenatide to a thiazolidinedione in sub-optimally controlled type 2 diabetes. A randomized trial. Ann Intern Med 2007;146:477-85.

73. Zinman B, Gerich J, Buse J, et al. Efficacy and safety of the human glucagon-like peptide-1 analog liraglutide in combination with metformin and thiazolidinedione in patients with type 2 diabetes (LEAD-4 Met+TZD). Diabetes Care 2009;32:1224-30.

74. Gardiner SM, March JE, Kemp PA, et al. Autonomic nervous system-dependent and-independent cardiovascular effects of exendin-4 infusion in conscious rats. $\mathrm{Br} J$ Pharmacol 2008;154:60-71.

75. Griffioen KJ, Wan R, Okun E, et al. GLP-1 receptor stimulation depresses heart rate variability and inhibits neurotransmission to cardiac vagal neurons. Cardiovasc Res 2010;14:1-7. 